\title{
Conformal quantum mechanics \& the integrable spinning Fishnet
}

\section{Sergey Derkachov ${ }^{a}$ and Enrico Olivucci ${ }^{b}$}

${ }^{a}$ St. Petersburg Department of the Steklov Mathematical Institute of Russian Academy of Sciences, Fontanka 27, 191023 St. Petersburg, Russia

${ }^{b}$ Perimeter Institute for Theoretical Physics,

31 Caroline Street North, Waterloo, ON N2L2Y5, Canada

E-mail: derkach@pdmi.ras.ru, e.olivucci@gmail.com

ABSTRACT: In this paper we consider systems of quantum particles in the $4 d$ Euclidean space which enjoy conformal symmetry. The algebraic relations for conformal-invariant combinations of positions and momenta are used to construct a solution of the Yang-Baxter equation in the unitary irreducibile representations of the principal series $\Delta=2+i \nu$ for any left/right spins $\ell, \dot{\ell}$ of the particles. Such relations are interpreted in the language of Feynman diagrams as integral star-triangle identites between propagators of a conformal field theory. We prove the quantum integrability of a spin chain whose $k$-th site hosts a particle in the representation $\left(\Delta_{k}, \ell_{k}, \dot{\ell}_{k}\right)$ of the conformal group, realizing a spinning and inhomogeneous version of the quantum magnet used to describe the spectrum of the bi-scalar Fishnet theories [1]. For the special choice of particles in the scalar $(1,0,0)$ and fermionic $(3 / 2,1,0)$ representation the transfer matrices of the model are Bethe-Salpeter kernels for the double-scaling limit of specific two-point correlators in the $\gamma$-deformed $\mathcal{N}=4$ and $\mathcal{N}=2$ supersymmetric theories.

Keywords: Conformal Field Theory, Field Theories in Higher Dimensions, Higher Spin Symmetry, Lattice Integrable Models

ArXiv EPrint: 2103.01940 


\section{Contents}

1 Introduction 1

2 Conformal quantum mechanics and star-triangle relation 2

3 General SO(1,5) R-operator 10

4 Inhomogeneous spinning Fishnet 16

4.1 Integrable correlators in conformal field theories 22

5 Outlooks

\section{Introduction}

This work is based on the papers [2-5]; our motivation is to clarify their various interrelations and to deduce how their results apply to the most general setup. First of all we managed to reformulate the expression given in [3] for the intertwiners $S_{1}, S_{2}, S_{3}$ of unitary irreducible representations of $\mathrm{SO}(1,5)$, and thus the infinite-dimensional R-operator, in the form of symmetric traceless tensors of coordinates and momenta $[2,6]$. Ultimately, these achievements are based on the new star-triangle relation derived in [4]. On the operative level we developed an handful Feynman diagram notation of lines and dots where the algebraic calculations based on the star-triangle duality are realized as graphical operations, providing ground for an extension to spinning conformal fields of the techniques [7-11] (see also the software implementation $[12,13])$. Secondly, by the knowledge of the R-operator we can formulate a new quantum integrable model with conformal symmetry as a chain of particles in the space $\mathbb{R}^{4}$, each of them transforming in a given representations of the principal series [14]. The transfer matrices of the model are identified with graph-building operators of a planar Feynman integral with square-lattice topology and spinning propagators.

The organization of the paper is the following. In the section 2 we present a compact formula for the Fourier transformation of the general conformal propagator in the representation of scaling dimension $\Delta$ and spins $\ell / 2$ and $\dot{\ell} / 2$. Along with it we introduce the generalized star-triangle duality involving such propagators and we explain the diagrammatic notations. The duality allows to derive by few graphical steps two main interchange relations which are ubiquitous in the proofs of commutativity of the transfer matrices of the integrable chain. Section 3 is devoted to the construction of the R-operator for general principal series representations, and the proof of the corresponding Yang-Baxter relation is delivered in diagrammatic form. As a whole, the results of the first two sections contain the four-dimensional version of the $\operatorname{SL}(2, \mathbb{C})$ techniques developed in [15] for $2 d$ conformal quantum models. 
In section 4 we construct the integrable chain starting from its transfer matrices. We propose a factorization of the transfer matrices in the product of two commuting operators and use it to demonstrate the quantum integrability of the model. For specific choices of the representations of the chain's particles the integral kernel of the transfer matrix operator is the Bethe-Salpeter kernel for the planar two-point functions of various Fishnet/chiral conformal field theories [1, 16-19].

\section{Conformal quantum mechanics and star-triangle relation}

Let us consider a quantum particle with left/right spins $\frac{\ell}{2}$ and $\frac{\dot{\ell}}{2}$ moving in the $4 d$ Euclidean space. Its state is described by a wave function of the position $x=\left(x_{0}, x_{1}, x_{2}, x_{3}\right)$ with $\ell$ and $\dot{\ell}$ - symmetric spinor indices

$$
\Phi_{\mathbf{a} \dot{\mathbf{a}}}(x)=\Phi_{\left(a_{1} \ldots a_{\ell}\right)\left(\dot{a}_{1} \ldots \dot{a}_{\dot{\ell}}\right)}(x), \quad a_{i}, \dot{a}_{i} \in\{1,2\},
$$

where $\left(a_{1} \ldots a_{\ell}\right)$ means symmetrization $(\ldots a \ldots b \ldots)=(\ldots b \ldots a \ldots)$.

The wavefunctions (2.1) belong to the Hilbert space

$$
\mathbb{V} \simeq L^{2}\left(d^{4} x\right) \otimes \operatorname{Sym}_{\ell}\left[\mathbb{C}^{2}\right] \otimes \operatorname{Sym}_{\dot{\ell}}\left[\mathbb{C}^{2}\right],
$$

with $\operatorname{Sym}_{\ell}\left[\mathbb{C}^{2}\right] \subset\left(\mathbb{C}^{2}\right)^{\otimes \ell}$ the space of complex symmetric spinors $u_{\mathbf{a}}=u_{\left(a_{1}, \ldots, a_{\ell}\right)}$. The scalar product on (2.2) is inherited from the standard ones defined on its factors, that is

$$
\langle F, G\rangle_{\mathbb{V}}=\int d^{4} x\langle F(x) \mid G(x)\rangle=\int d^{4} x\left(F^{*}\right)^{\mathbf{a} \dot{\mathbf{a}}}(x) G_{\mathbf{a} \dot{\mathbf{a}}}(x) .
$$

The spinors in paper have lower indices $\alpha_{a}$ and $\beta_{\dot{a}}$ and the index raising operation is defined as complex conjugation $\alpha^{a}=\left(\alpha_{a}\right)^{*}=\bar{\alpha}_{a}$ and $\beta^{\dot{a}}=\left(\beta_{\dot{a}}\right)^{*}=\bar{\beta}_{\dot{a}}$ and the pairing between spinors is the standard scalar product in $\mathbb{C}^{2}$

$$
\left\langle\alpha \mid \alpha^{\prime}\right\rangle=\alpha^{a} \alpha_{a}^{\prime}=\bar{\alpha}_{a} \alpha_{a}^{\prime}, \quad\left\langle\beta^{\prime} \mid \beta\right\rangle=\beta^{\prime \dot{a}} \beta_{\dot{a}}=\bar{\beta}_{\dot{a}}^{\prime} \beta_{\dot{a}} .
$$

A rotation of the Euclidean space $x^{\mu} \mapsto \Lambda_{\nu}^{\mu} x^{\nu}$ is represented on the symmetric spinors according to the decomposition $\mathrm{SO}(4) \simeq \mathrm{SU}(2) \times \mathrm{SU}(2)$, by two matrices $U, V$ rotating the dotted/undotted indices

$$
u_{\mathbf{a}} \mapsto u_{\mathbf{a}}^{\prime}=[U]_{\mathbf{a}}^{\mathbf{b}} u_{\mathbf{b}}, \quad v_{\dot{\mathbf{a}}} \mapsto v_{\dot{\mathbf{a}}}^{\prime}=[V]_{\dot{\mathbf{a}}}^{\dot{\mathbf{b}}} v_{\dot{\mathbf{b}}},
$$

and the following notations will be frequently used throughout the text

$$
\begin{aligned}
& \mathbf{a} \equiv\left(a_{1}, \ldots, a_{\ell}\right), \quad \dot{\mathbf{a}} \equiv\left(\dot{a}_{1}, \ldots, \dot{a}_{\dot{\ell}}\right), \quad a_{k}, \dot{a}_{k} \in\{1,2\}, \\
& u_{\mathbf{a}}=u_{\left(a_{1} \ldots a_{\ell}\right)}, \quad v_{\dot{\mathbf{a}}}=v_{\left(\dot{a}_{1} \ldots \dot{a}_{\ell}\right)}, \quad[U]_{\mathbf{a}}^{\mathbf{b}}=U_{\left(a_{1}\right.}^{\left(b_{1}\right.} \cdots U_{\left.a_{\ell}\right)}^{\left.b_{\ell}\right)} .
\end{aligned}
$$

Alternatively we will use the compact notation $[U]^{\ell}$ which specifies the spin number $\ell$ without explicit spinor indices

$$
\left([U]^{\ell}\right)_{\mathbf{a}}^{\mathbf{b}}=U_{\left(a_{1}\right.}^{\left(b_{1}\right.} \cdots U_{\left.a_{\ell}\right)}^{\left.b_{\ell}\right)}
$$


We are interested in quantum systems with conformal symmetry, that is in particles evolving under the action of an Hamiltonian operator $\mathcal{H}$ which is invariant under conformal transformation

$$
x^{\mu} \mapsto y^{\mu}(x), \quad \frac{\partial y^{\mu}(x)}{\partial x^{\kappa}} \frac{\partial y_{\nu}(x)}{\partial x_{\kappa}}=\Lambda(y) \delta_{\nu}^{\mu} .
$$

Examples of such systems in a $d=2$ dimensional spacetime are the $\operatorname{SL}(2, \mathbb{C})$ integrable spin chains introduced by Lipatov to describe scattering amplitudes of QCD gluons at high energy [20-22]. Recently, higher dimensional $(d>2)$ versions of conformal spin chains have attracted attention in the realm of Fishnet conformal field theories [1, 4, 16, 23-26], as they describe the Feynman integrals of the theory at any coupling and therefore the anomalous dimensions of operators at finite coupling. In a conformal invariant system the eigenfunctions of the model should transform under an irreducible unitary representation of the conformal group. Hence, we study wavefunctions transforming as

$$
x^{\mu} \mapsto y^{\mu}(x), \quad \Phi_{\mathbf{a} \dot{\mathbf{a}}}(x) \mapsto \Phi^{\prime} \mathbf{a \dot { a }}(x)=\Lambda(y)^{\Delta}[U]_{\mathbf{a}}^{\mathbf{b}}[V]_{\dot{\mathbf{a}}}^{\dot{\mathbf{b}}} \Phi_{\mathbf{b} \dot{\mathbf{b}}}(y),
$$

where $U_{a}^{b}$ and $V_{\dot{a}}^{\dot{b}}$ are two $\mathrm{SU}(2)$ matrices that realize the spinor representation of $\mathrm{SO}(4)$ rotations. The dotted and un-dotted indices distinguish spinors that are transformed according to representations of two different copies of $\mathrm{SU}(2)$.

Without loss of generality we can restrict ourselves to the representations of the principal series, that means $\Delta=2+i \nu$ with $\nu$ a real number. ${ }^{1}$ A scale-invariant combination of the position operator $x^{\mu}$ and momentum operator $\hat{p}_{\mu}=-i \partial_{\mu}$ satisfies the remarkable duality $[2,6]$

$$
\hat{p}^{2 u} x^{2(u+v)} \hat{p}^{2 v}=x^{2 v} \hat{p}^{2(u+v)} x^{2 u},
$$

where the operator $\hat{p}^{2 u}$ is understood as an integral operator

$$
\hat{p}^{2 u} f(x)=\frac{1}{a(u)} \int d^{4} y \frac{f(y)}{(x-y)^{2(u+2)}} .
$$

Note that we shall consider the generic situation $u \in \mathbb{C}$ and understand all similar integrals as an analytic continuation in $u$ from the convergence domain [27].

The representation (2.11) follows from the formula for the Fourier transformation of a power function

$$
\int d^{4} x \frac{e^{i p x}}{x^{2(u+2)}}=a(u) p^{2 u}, a(u)=\frac{\pi^{2} \Gamma(-u)}{4^{u} \Gamma(u+2)},
$$

where $\Gamma(u)$ is the Euler gamma-function.

According to (2.11) the equation (2.10) has a straightforward interpretation in the language of Feynman diagrams as a star-triangle identity as illustrated in figure 1. Indeed, (2.10) is an identity between two integral operators and the application of both sides of the equation to the delta-function $\delta^{(4)}(x-z)$ delivers an equality between the corresponding kernels

$$
\int \frac{d^{4} y}{(x-y)^{2(u+2)} y^{-2(u+v)}(y-z)^{2(v+2)}}=\frac{a(u) a(v)}{a(u+v)} \frac{1}{x^{-2 v}(x-z)^{2(u+v+2)} z^{-2 u}},
$$

\footnotetext{
${ }^{1}$ The unitary irreducible representations of $\mathrm{SO}(1,5)$ — as explained in [14] — would include also the complementary series, for which $0<\Delta<4$. Our results throughout the paper can be extended to this class of representations by an analytic continuation of $\nu$ to the imaginary segment $i[-2,2]$.
} 

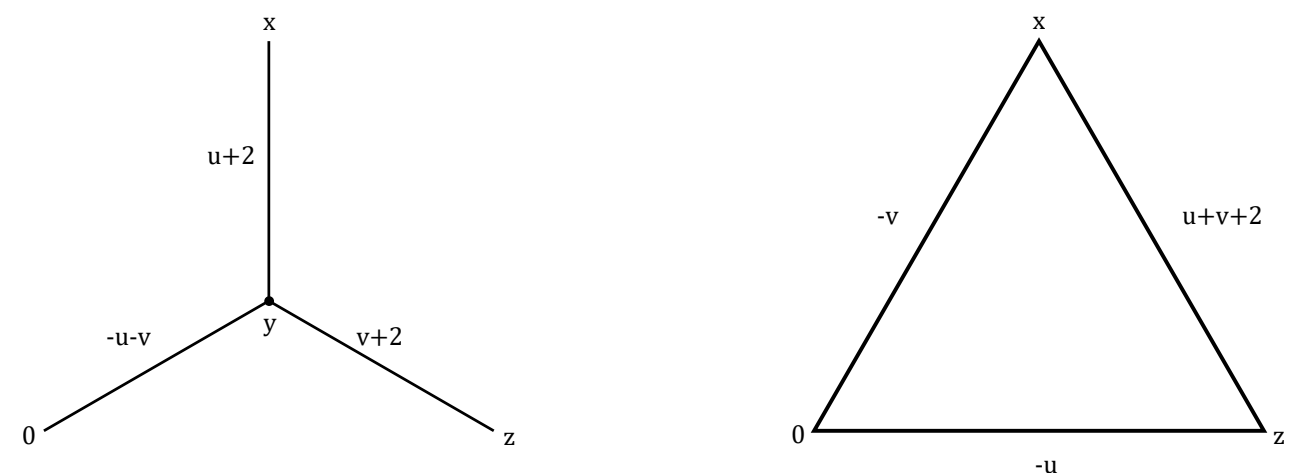

Figure 1. Left: convolution of three scalar propagators corresponding to the l.h.s. of the duality (2.13). Each solid segment is $1 / x_{i j}^{2}$ to the power written next to it, where $x_{i}$ and $x_{j}$ are the endpoints of the segment. Right: product of three propagators corresponding to the r.h.s. of (2.13) modulo a multiplicative pre-factor.

that in terms of Feynman diagrams relates a vertex of three propagators whose scaling dimensions sum up to $d=4$ - the star integral - with the product of three propagators between the vertices of the star - the triangle. In the following we generalize the equality (2.10) to the case of spinning particles $\ell, \dot{\ell} \neq 0$. Therefore, a scalar propagators $x^{2 u}$ of a field of dimension $\Delta=-u$ is replaced by the propagator of a conformal field in the representation $(\Delta=-u, \ell, \dot{\ell})$, that stripped of all constants reads [28]

$$
G_{\ell \dot{\ell}}(u, x)=x^{2 u}[\overline{\mathbf{x}}]^{\ell}[\mathbf{x}]^{\dot{\ell}}, \quad \mathbf{x}=\boldsymbol{\sigma}_{\mu} \frac{x^{\mu}}{\left(x^{2}\right)^{\frac{1}{2}}}, \quad \overline{\mathbf{x}}=\overline{\boldsymbol{\sigma}}_{\mu} \frac{x^{\mu}}{\left(x^{2}\right)^{\frac{1}{2}}} .
$$

Here the matrices $\boldsymbol{\sigma}_{\mu}$ and $\overline{\boldsymbol{\sigma}}_{\mu}$ are defined in terms of the standard Pauli matrices as $\boldsymbol{\sigma}_{k}=$ $i \sigma_{k}, \overline{\boldsymbol{\sigma}}_{k}=-i \sigma_{k}$ for $k=1,2,3$ and $\boldsymbol{\sigma}_{0}=\boldsymbol{\sigma}_{0}=\mathbb{1}$, so that they satisfy the Clifford algebra

$$
\boldsymbol{\sigma}_{\mu} \overline{\boldsymbol{\sigma}}_{\nu}+\boldsymbol{\sigma}_{\nu} \overline{\boldsymbol{\sigma}}_{\mu}=2 \delta_{\mu \nu} .
$$

Everywhere in the paper we work with Euclidean metric and it is not useful for our purposes to distinguish carefully the upper and lower tensor indices like $\mu, \nu$. For the sake of simplicity for the cumbersome formulae we adopt the following notation in the paper for spinor indices $\boldsymbol{\sigma}_{\mu}=\left\|\left(\boldsymbol{\sigma}_{\mu}\right)_{a}^{\dot{a}}\right\|$ and $\overline{\boldsymbol{\sigma}}_{\mu}=\left\|\left(\overline{\boldsymbol{\sigma}}_{\mu}\right)_{\dot{a}}^{a}\right\|$ so that (2.14) in explicit notations with all spinor indices has the following form

$$
G_{\ell \dot{\ell}}(u, x)_{\dot{\mathbf{a}} \mathbf{a}}^{\mathbf{b}} \dot{\mathbf{b}}=x^{2 u}[\overline{\mathbf{x}}]_{\dot{\mathbf{a}}}^{\mathbf{b}}[\mathbf{x}]_{\mathbf{a}}^{\dot{\mathbf{b}}} .
$$

Of course the same generalization of $\hat{p}^{2 u} \rightarrow G_{\ell \dot{\ell}}(u, p)$ can be done for the momentum operator. The main difference w.r.t. the case of the scalar propagators is that the Fourier transformation of (2.16) involves a mixing of spinorial indices:

$$
\int d^{4} x e^{i p x} \frac{[\overline{\mathbf{x}}]^{\ell}[\mathbf{x}]^{\dot{\ell}}}{x^{2(u+2)}}=a_{\ell \dot{\ell}}(u) \hat{p}^{2 u}[\overline{\mathbf{p}}]^{\ell} \mathbf{R}_{\ell \dot{\ell}}(u)[\mathbf{p}]^{\dot{\ell}},
$$

where the constant $a_{\ell \dot{\ell}}(u)$ is a generalization of the constant $a(u)$ to general non-zero spins $\ell, \dot{\ell}$

$$
a_{\ell \dot{\ell}}(u)=\frac{(-i)^{\ell+\dot{\ell}} \pi^{2} \Gamma\left(-u-\frac{\ell+\dot{\ell}}{2}\right) \Gamma\left(u+\frac{\ell+\dot{\ell}}{2}+1\right)}{4^{u}\left(u+\frac{\ell+\dot{\ell}}{2}+1\right) \Gamma\left(u+1+\frac{\ell-\dot{\ell}}{2}\right) \Gamma\left(u+1+\frac{\dot{\ell}-\ell}{2}\right)} .
$$




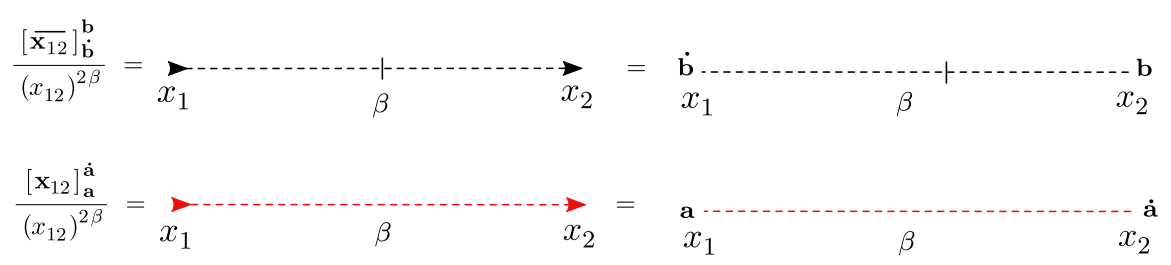

Figure 2. Graphic representation of the propagator $G_{\ell \dot{\ell}}\left(u, x_{1}-x_{2}\right)$ of (2.16) with conformal dimension $\beta=-u$ and spins $(\ell, \dot{\ell})=(0, \dot{\ell})-$ first row - or $(\ell, \dot{\ell})=(\ell, 0)-$ second row. The dashed lines stand for the spinorial structures in $(2.16)$, the one with the bar denoting the matrix $\overline{\mathbf{x}}$. The arrows denote the flow of spinor indices.

The matrix $\mathbf{R}_{n m}(u)$ appearing in the r.h.s. of (2.17) is the compact notation for

$$
\mathbf{R}_{\mathbf{a c}}^{\mathbf{b d}}(u)=\mathbf{R}_{\left(a_{1} \ldots a_{n}\right)\left(c_{1} \ldots c_{m}\right)}^{\left(b_{1} \ldots b_{n}\right)\left(d_{1} \ldots d_{m}\right)}(u)
$$

which is a solution of the Yang-Baxter equation (YBE) on the space of $n$ - and $m$-symmetric spinors

$$
\mathbf{R}_{n m}(u-v) \mathbf{R}_{n \ell}(u) \mathbf{R}_{m \ell}(u)=\mathbf{R}_{m \ell}(v) \mathbf{R}_{n \ell}(u) \mathbf{R}_{n m}(u-v) .
$$

It can be obtained by the fusion procedure $[29,30]$ starting from the well-known solution in the defining representation $n=m=1$

$$
\mathbf{R}_{a c}^{b d}(u)=\frac{1}{u+1}\left(u \delta_{a}^{b} \delta_{c}^{d}+\delta_{a}^{d} \delta_{c}^{b}\right): \mathbb{C}^{2} \otimes \mathbb{C}^{2} \longrightarrow \mathbb{C}^{2} \otimes \mathbb{C}^{2} .
$$

and the final expression is given by (C.12)-(C.14) of the paper [5]. Besides the YBE, the $\mathbf{R}$-matrix satisfies the parity relation $\mathbf{R}_{n m}(u) \mathbf{R}_{n m}(-u)=\mathbb{1}$, or in explicit form

$$
\mathbf{R}_{\mathbf{a}_{1} \mathbf{a}_{2}}^{\mathbf{c}_{1} \mathbf{c}_{2}}(u) \mathbf{R}_{\mathbf{c}_{1} \mathbf{c}_{2}}^{\mathbf{b}_{1} \mathbf{b}_{2}}(-u)=\delta_{\mathbf{a}_{1}}^{\mathbf{b}_{1}} \delta_{\mathbf{a}_{2}}^{\mathbf{b}_{2}}
$$

and the crossing symmetry $\mathbf{R}_{n m}^{t}(u) \mathbf{R}_{n m}^{t}(-u-2)=r_{n m}(u) \mathbb{1}$,

$$
\mathbf{R}_{\mathbf{c}_{1} \mathbf{a}_{2}}^{\mathbf{a}_{1} \mathbf{c}_{2}}(u) \mathbf{R}_{\mathbf{b}_{1} \mathbf{c}_{2}}^{\mathbf{c}_{1} \mathbf{b}_{2}}(-u-2)=r_{n m}(u) \delta_{\mathbf{b}_{1}}^{\mathbf{a}_{1}} \delta_{\mathbf{a}_{2}}^{\mathbf{b}_{2}}
$$

with the scalar factor

$$
r_{n m}(u)=\frac{\left(u+\frac{n+m}{2}+1\right)}{\left(u+\frac{n-m}{2}+1\right)} \frac{\left(-u+\frac{n+m}{2}-1\right)}{\left(-u+\frac{n-m}{2}-1\right)},
$$

and $t$ means transposition in the space of $n$-symmetric spinors. The crossing symmetry (2.23) allows to rewrite the equality (2.17) in the equivalent form

$$
r_{\ell \dot{\ell}}(u) a_{\ell \dot{\ell}}(u) \hat{p}^{2 u}[\overline{\mathbf{p}}]^{\ell}[\mathbf{p}]^{\dot{\ell}}=\int d^{4} x e^{i p x} \frac{[\overline{\mathbf{x}}]^{\ell} \mathbf{R}_{\ell \dot{\ell}}(-u-2)[\mathbf{x}]^{\dot{\ell}}}{x^{2(u+2)}} .
$$

In terms of Feynman diagrams we represent the spinning propagators (2.16) by a double dashed line, see figures 2, 4. Each line stands for the spinor structure $[\mathbf{x}]$ or $[\overline{\mathbf{x}}]$, as in figure 2, and we use arrows to indicate the flow of spinor indices. In fact, the matrices $\mathbf{R}(u)$ and $\overline{\mathbf{R}}(u)$ differ only for the type of indices (un-dotted/dotted) but their 


$$
\begin{aligned}
& \frac{\left[\mathbf{x}_{12} \overline{\mathbf{x}_{23}}\right]_{\mathbf{a}}^{\mathbf{b}}}{\left(x_{12}\right)^{2 \beta}\left(x_{13}\right)^{2 \gamma}}=\frac{\left[\mathbf{x}_{12}\right]_{\mathbf{a}}^{\dot{\mathbf{c}}}\left[\overline{\mathbf{x}_{23}}\right]_{\dot{\mathbf{c}}}^{\mathbf{b}}}{\left(x_{12}\right)^{2 \beta}\left(x_{13}\right)^{2 \gamma}}=
\end{aligned}
$$

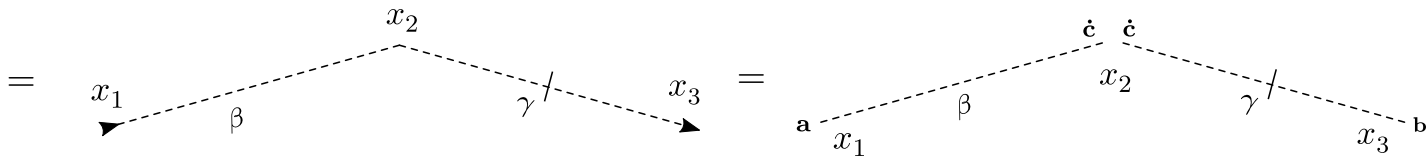

Figure 3. Graphic representation of the product of two propagators respect to their spinor structure. The arrows denote the order of the matrices $[\mathbf{x}]$ and $[\overline{\mathbf{x}}]$ in the product.

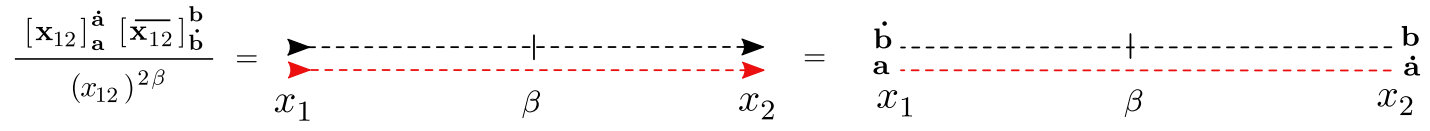

Figure 4. Graphic representation of the propagator $G_{\ell \dot{\ell}}\left(u, x_{1}-x_{2}\right)$ of (2.16) with conformal dimension $\beta=-u$ and generic spins $\ell, \dot{\ell}$. The two dashed lines stand for the spinorial structures in (2.16), the one with the bar denoting the matrix $\overline{\mathbf{x}}$. The arrows denote the flow of spinor indices, as made explicit by the r.h.s.

$$
\begin{aligned}
& \frac{\left[\overline{\mathbf{x}}_{12}\right]_{\dot{\mathbf{b}}}^{\mathbf{e}} \mathbf{R}_{\mathbf{e a}}^{\mathbf{b c}}(u)\left[\mathbf{x}_{12}\right]_{\mathbf{c}}^{\dot{\mathbf{a}}}}{\left(x_{12}\right)^{2 \beta}}=\underset{x_{1}}{-}
\end{aligned}
$$

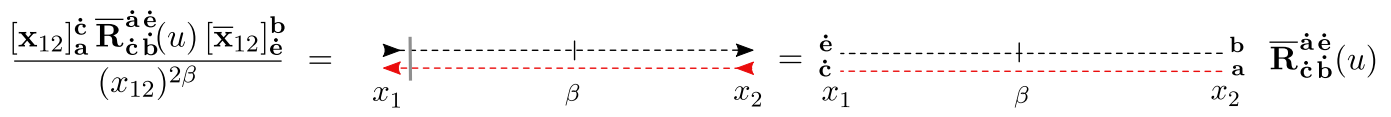

Figure 5. Graphic representation of the propagator $S_{\ell \dot{\ell}}\left(u, x_{1}-x_{2}\right)$ of $(2.26)$. The two dashed lines stand for the spinorial structures in (2.16), and the mixing matrix $\mathbf{R}$ (or $\overline{\mathbf{R}}$ ) is inserted along their structure as a solid grey line. On the right we show the explicit spinor structure of $S_{\ell \dot{\ell}}\left(u, x_{1}-x_{2}\right)$, corresponding to the compact notation on the left.

matrix elements are identical, thus we'll often omit to specify the over-lined notation in the following. Moreover, pair of lines of the type appearing in the notation of figure 4 are always assumed to be one of type $[\mathbf{x}]$ and one of type $[\overline{\mathbf{x}}]$, even when not specified by the graphical notation. Similarly, two convoluted lines as in figure 3 must be always $[\mathbf{x}][\overline{\mathbf{x}}]$ either $[\overline{\mathbf{x}}][\mathbf{x}]$. All the formulas that we write in the paper are actually invariant under the exchange $\boldsymbol{\sigma} \leftrightarrow \overline{\boldsymbol{\sigma}}$.

Let us state the rules

$$
[\mathbf{x}]_{\mathbf{a}}^{\dot{\mathbf{c}}} \overline{\mathbf{R}}_{\dot{\mathbf{a}} \dot{\mathbf{c}}}^{\dot{\mathbf{d}} \dot{\mathbf{b}}}(u)[\overline{\mathbf{x}}]_{\dot{\mathbf{d}}}^{\mathbf{b}}=[\overline{\mathbf{x}}]_{\dot{\mathbf{a}}}^{\mathbf{c}} \mathbf{R}_{\mathbf{c a}}^{\mathbf{b d}}(u)[\mathbf{x}]_{\mathbf{d}}^{\dot{\mathbf{b}}}, \quad \overline{\mathbf{R}_{\dot{\mathbf{a}} \dot{\mathbf{c}}}} \dot{\dot{d}} \dot{\mathbf{b}}(u)[\overline{\mathbf{x}}]_{\dot{\mathbf{d}}}^{\mathbf{a}}[\overline{\mathbf{x}}]_{\dot{\mathbf{b}}}^{\mathbf{c}}=[\overline{\mathbf{x}}]_{\dot{\mathbf{a}}}^{\mathbf{d}}[\overline{\mathbf{x}}]_{\dot{\mathbf{c}}}^{\mathbf{b}} \mathbf{R}_{\mathbf{d} \mathbf{b}}^{\mathbf{a c}}(u)
$$

which are often tacitly used in computations to move the matrix $\mathbf{R}(u)$ along paired spinorial structures. In the diagrammatic notation of figure 5 they amount to the equalities:
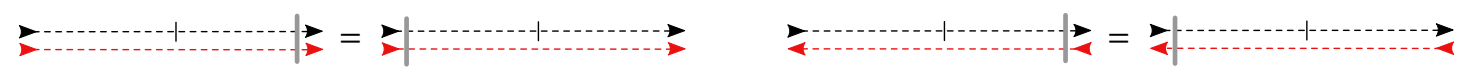

With the same notation, we can represent the properties of the $\mathbf{R}$-matrix, as in figure 6 .

In the following we will write the spin numbers $n, m$ in the notation $\mathbf{R}_{n m}(u)$ only when using the compact form (2.7), while we will drop them when the spinor indices are written 


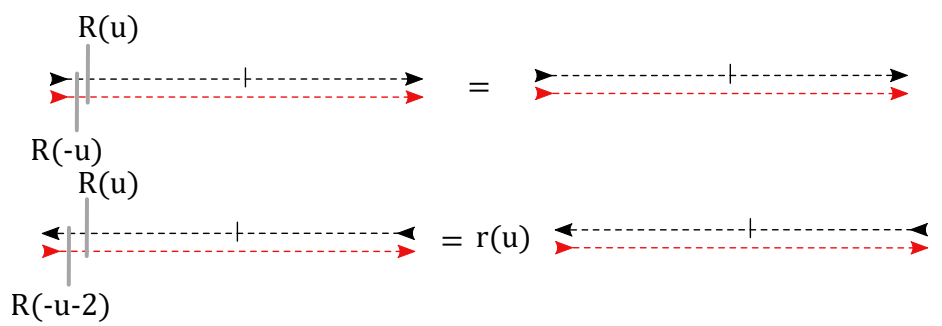

Figure 6. $U p$ : parity property of the matrix $\mathbf{R}_{m n}(u)$. The product of two matrices with opposite arguments along two spinorial structure with the same orientation cancels. Down: crossing symmetry of the matrix $\mathbf{R}_{m n}(u)$. The two matrices act along two spinor structures flowing in the opposite directions, according to the arrows, so their product is transposed in one of the two spinor spaces.

explicitly. Let us define the matrix function

$$
S_{\ell \dot{\ell}}(u, x)=x^{2 u}[\overline{\mathbf{x}}]^{\ell} \mathbf{R}_{\ell \dot{\ell}}(u)[\mathbf{x}]^{\dot{\ell}},
$$

which is related to $G_{\ell \dot{\ell}}(-u-2, p)(2.14)$ according to $(2.24)$.

Similarly, using $(2.17)$ it is possible to realize the operator $S_{\ell \dot{\ell}}(u, \hat{p})$ as an integral operator with the kernel $G_{\ell \dot{\ell}}(-u-2, x-y)$

$$
\hat{p}^{2 u}[\overline{\mathbf{p}}]^{\ell} \mathbf{R}_{\ell \dot{\ell}}(u)[\mathbf{p}]^{\dot{\ell}} \Phi(x)=\frac{1}{a_{\ell \dot{\ell}}(u)} \int d^{4} y \frac{[\overline{\mathbf{x}-\mathbf{y}}]^{\ell}[\mathbf{x}-\mathbf{y}]^{\dot{\ell}}}{(x-y)^{2(u+2)}} \Phi(y) .
$$

The generalization of the algebraic relation (2.10) to spinning representations is achieved by replacing the operators $\hat{p}^{2 u}$ and $x^{2 u}$ with their spinning counterparts $S(u, p)$ and $S(u, x)$ as follows

$$
S_{\dot{\ell} m}(u, p) S_{m \ell}(u+v, x) S_{\dot{\ell}(}(v, p)=S_{\dot{\ell} \ell}(v, x) S_{\ell m}(u+v, p) S_{m \dot{\ell}}(u, x),
$$

or more explicitly:

$$
\begin{aligned}
& \hat{p}^{2 u}[\overline{\mathbf{p}}]^{\dot{\ell}} \mathbf{R}_{m \dot{\ell}}(u)[\mathbf{p}]^{m} x^{2(u+v)}[\overline{\mathbf{x}}]^{m} \mathbf{R}_{m \ell}(u+v)[\mathbf{x}]^{\ell} \hat{p}^{2 v}[\overline{\mathbf{p}}]^{\ell} \mathbf{R}_{\ell \dot{\ell}}(v)[\mathbf{p}]^{\dot{\ell}} \\
& =x^{2 v}[\overline{\mathbf{x}}]^{\dot{\ell}} \mathbf{R}_{\ell \dot{\ell}}(v)[\mathbf{x}]^{\ell} \hat{p}^{2(u+v)}[\overline{\mathbf{p}}]^{\ell} \mathbf{R}_{m \ell}(u+v)[\mathbf{p}]^{m} x^{2 u}[\overline{\mathbf{x}}]^{m} \mathbf{R}_{m \dot{\ell}}(u)[\mathbf{x}]^{\dot{\ell}} .
\end{aligned}
$$

The propagators involved in the spinning star-triangle duality (2.29) carry spin indices in the spaces

$$
\operatorname{Sym}_{m}\left[\mathbb{C}^{2}\right] \otimes \operatorname{Sym}_{\ell}\left[\mathbb{C}^{2}\right] \otimes \operatorname{Sym}_{\dot{\ell}}\left[\mathbb{C}^{2}\right],
$$

which are pair-wise mixed on both the star and the triangle sides of the identity by the R-matrix. Applying the both sides of (2.29) to the delta-function $\delta^{(4)}(x-z)$ we obtain the following identity for integral kernels

$$
\begin{aligned}
\int d^{4} y \frac{[\overline{\mathbf{x}-\mathbf{y}}]^{\dot{\ell}}[\mathbf{x}-\mathbf{y}]^{m}}{(x-y)^{2(u+2)}} \frac{[\overline{\mathbf{y}}]^{m} \mathbf{R}_{m \ell}(u+v)[\mathbf{y}]^{\ell}}{y^{-2(u+v)}} \frac{[\overline{\mathbf{y}-\mathbf{z}}]^{\ell}[\mathbf{y}-\mathbf{z}]^{\dot{\ell}}}{(y-z)^{2(v+2)}}= \\
=\frac{a_{\dot{\ell} m}(u) a_{\dot{\ell}}(v)}{a_{\ell m}(u+v)} \frac{[\overline{\mathbf{x}}]^{\dot{\ell}} \mathbf{R}_{\ell \dot{\ell}}(v)[\mathbf{x}]^{\ell}}{x^{-2 v}} \frac{[\overline{\mathbf{x}-\mathbf{z}}]^{\ell}[\mathbf{x}-\mathbf{z}]^{m}}{(x-z)^{2(u+v+2)}} \frac{[\overline{\mathbf{z}}]^{m} \mathbf{R}_{m \dot{\ell}}(u)[\mathbf{z}]^{\dot{\ell}}}{z^{-2 u}} .
\end{aligned}
$$



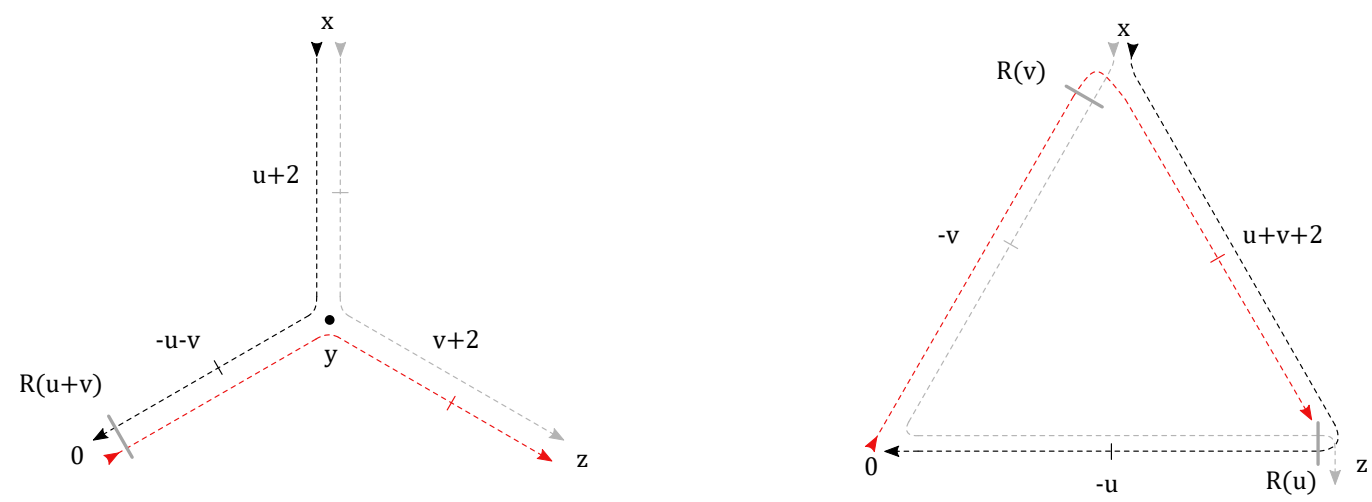

Figure 7. Left: diagrammatic form of the l.h.s. (2.32) of the star-triangle duality as a star of propagators. Right: diagrammatic form of the r.h.s. (2.33) of the star-triangle duality as a triangle of propagators. The spinor indices are mixed by the SU(2) fused R-matrices that are contracted with the matrices $\boldsymbol{\sigma}, \overline{\boldsymbol{\sigma}}$ appearing in the definition (2.14), and their position is denoted by grey solid lines. The black dot in the star is the integrated point $y$. The letter adjacent to each segment is the scaling dimension of the propagator.

The 1.h.s. of (2.31) is the integrated vertex $y$ of three propagators. Each of them in one the irreps $(2-v, \ell, \dot{\ell}),(u+v, m, \ell)$ and $(2-u, \dot{\ell}, m)$, and it is represented on the left of figure 7 . In explicit form it reads

$$
\mathbf{R}(u+v)_{\mathbf{s a}}^{\mathbf{d} \mathbf{s}^{\prime}} \int d^{4} y \frac{[(\mathbf{x}-\mathbf{y}) \overline{\mathbf{y}}]_{\mathbf{b}}^{\mathbf{s}}[\mathbf{y}(\overline{\mathbf{y}-\mathbf{z}})]_{\mathbf{s}^{\prime}}^{\mathbf{c}}[(\overline{\mathbf{x}-\mathbf{y}})(\mathbf{y}-\mathbf{z})]_{\dot{\mathbf{a}}}^{\dot{\mathbf{d}}}}{(x-y)^{2(u+2)} y^{2(-u-v)}(y-z)^{2(v+2)}} .
$$

Repeated spinorial indices are contracted, and the indices in $\operatorname{Sym}_{\ell} \otimes \mathrm{Sym}_{m}$ are mixed by the matrix $\mathbf{R}_{\ell m}(u+v)$. The star vertex is scale invariant as the sum of scaling dimensions of the propagators is equal to the dimension $d=4$ of the space and is proportional to a product of propagators in the irreps $(u, \ell, \dot{\ell}),(2-u-v, m, \ell)$ and $(v, \dot{\ell}, m)$, forming the triangle in the right of figure 7. On the r.h.s. of figure 7 the indices in $\mathrm{Sym}_{\dot{\ell}} \otimes \mathrm{Sym}_{m}$ and $\operatorname{Sym}_{\ell} \otimes \operatorname{Sym}_{\dot{\ell}}$ are mixed pairwise by the matrices $\mathbf{R}_{m \dot{\ell}}(u)$ and $\mathbf{R}_{\ell \dot{\ell}}(v)$

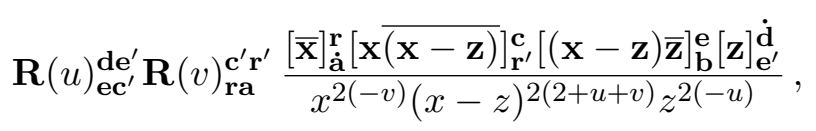

We can cast the identity in a more convenient form - depicted in figure 8 - where the mixing of spinors is removed from the l.h.s. of (2.31), moving the matrix $\mathbf{R}(u+v)$ to the r.h.s. by means of crossing symmetry (2.23). Indeed, contracting (2.32) and (2.33) with the matrix $\mathbf{R}(-u-v-2)_{\mathbf{d a}^{\prime}}^{\mathbf{d}^{\prime}}$ one cancels any mixing on the l.h.s. of the equation. After relabeling $\mathbf{a}^{\prime} \rightarrow \mathbf{a}, \mathbf{d}^{\prime} \rightarrow \mathbf{d}$ the star integral takes the form

$$
\int d^{4} y \frac{[(\mathbf{x}-\mathbf{y}) \overline{\mathbf{y}}]_{\mathbf{b}}^{\mathbf{d}}[\mathbf{y}(\overline{\mathbf{y}-\mathbf{z}})]_{\mathbf{a}}^{\mathbf{c}}[(\overline{\mathbf{x}-\mathbf{y}})(\mathbf{y}-\mathbf{z})]_{\mathbf{a}}^{\dot{\mathbf{d}}}}{(x-y)^{2(u+2)} y^{2(-u-v)}(y-z)^{2(v+2)}}
$$

while the triangle on the r.h.s. of the equation is

$$
\frac{a_{\dot{\ell} m}(u) a_{\ell \dot{\ell}}(v)}{r_{m \ell}(u+v) a_{\ell m}(u+v)} \mathbf{R}(-u-v-2)_{\mathbf{s a}}^{\mathbf{d s}^{\prime}} \mathbf{R}(u)_{\mathbf{e c}^{\prime}}^{\mathbf{s e}^{\prime}} \mathbf{R}(v)_{\mathbf{r s}^{\prime}}^{\mathbf{c}^{\prime} \mathbf{r}^{\prime}} \frac{[\overline{\mathbf{x}}]_{\dot{\mathbf{a}}}^{\mathbf{r}}[\mathbf{x} \overline{(\mathbf{x}-\mathbf{z})}]_{\mathbf{r}^{\prime}}^{\mathbf{c}}[(\mathbf{x}-\mathbf{z}) \overline{\mathbf{z}}]_{\mathbf{b}}^{\mathbf{e}}[\mathbf{z}]_{\mathbf{e}^{\prime}}^{\dot{\mathbf{d}}}}{x^{2(-v)}(x-z)^{2(2+u+v)} z^{2(-u)}}
$$



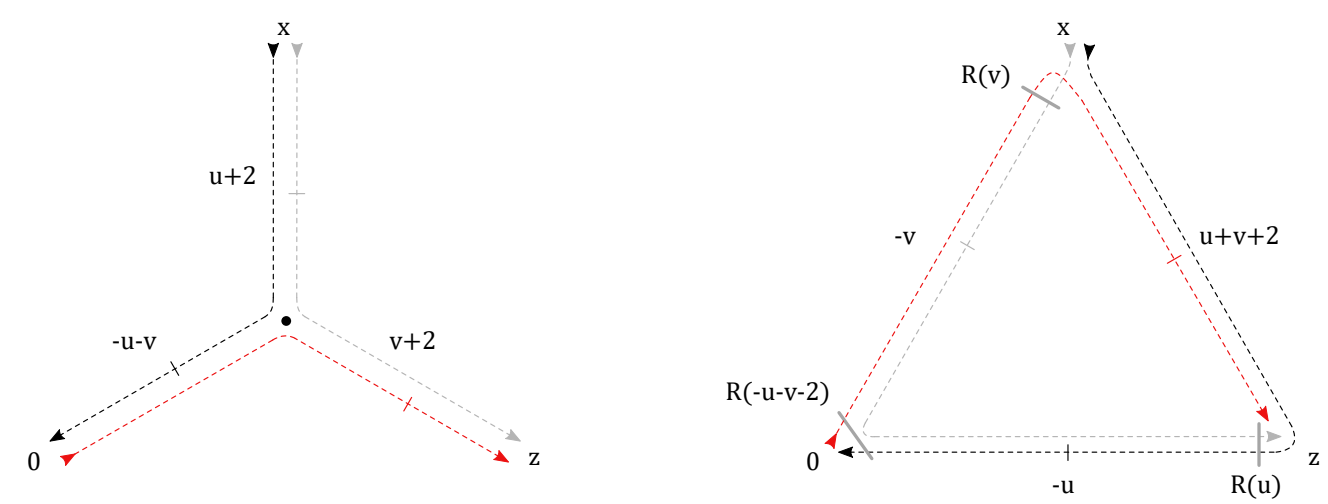

Figure 8. Diagrammatic representation of the star-triangle duality $(2.34)=(2.35)$. Here the star of conformal propagators (l.h.s.) does not contain any mixing of spinors, which happens only in the triangle (r.h.s.). The arrows denote the order in the matrix products of $\boldsymbol{\sigma}, \overline{\boldsymbol{\sigma}}$. Grey solid lines denote $\mathbf{R}$ matrices, and the black dot in the star is the integration point. The scaling dimension of each propagator is written next to it.

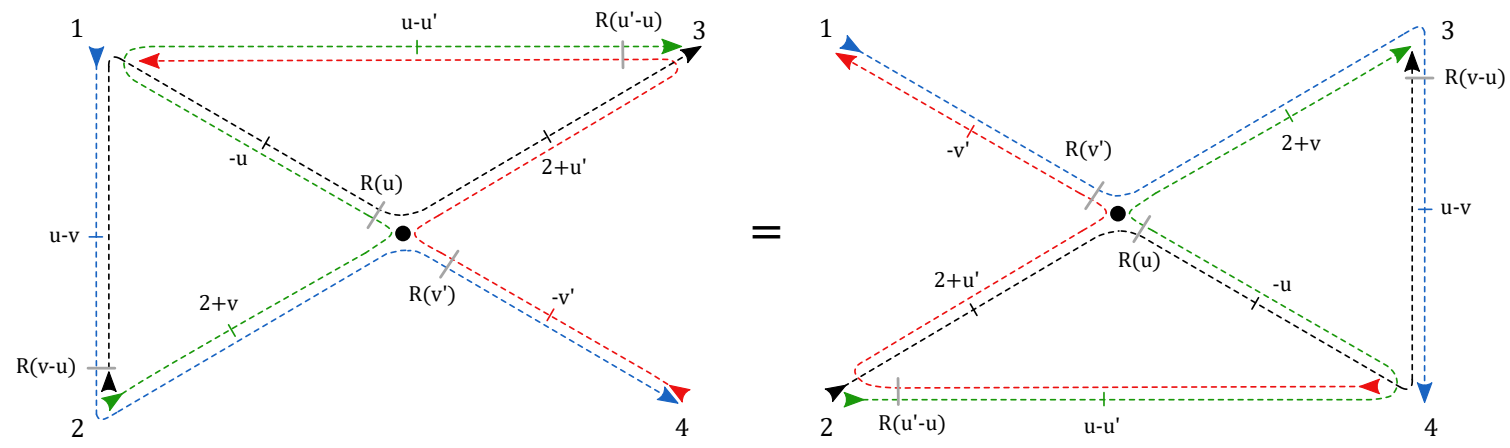

Figure 9. Diagrammatic form of the interchange relation I.

In this form, illustrated in figure 8 , the star-triangle identity relates a conformal-invariant vertex of three spinning propagators of type $G(u, x)$ with the product of three propagators whose spinorial indices are mutually mixed by the $\mathbf{R}$-matrix.

Let us point out that the diagrammatic approach of figures 1-8 is strikingly simpler than the analytic one, which motivates our artistic efforts in this section. The involved structure of spinorial indices and their contractions, for instance in (2.31)-(2.35), is entirely contained in the notation of two oriented dashed lines and solid gray $\mathbf{R}$-matrix insertions. In fact, the diagrammatic approach is the most compact way to perform computations in conformal quantum mechanics without loss of rigour, hence in most of the following we will avoid any analytic formula in favour of graphical derivations. Among the integral identities that one can derive by means of (2.28) we present two which are highly frequent in the computations of the next sections, which we dub interchange relations. Both identities describe the transformation of a conformal-invariant vertex of four spinning propagators (see figure 19) by a reshuffling of scaling dimensions and spins of the propagators. The first identity holds under the constraint $u+u^{\prime}=v+v^{\prime}$ and has the diagrammatic form: where the equality between the two diagrams is exact, i.e. the proportionality factor is 1 . 


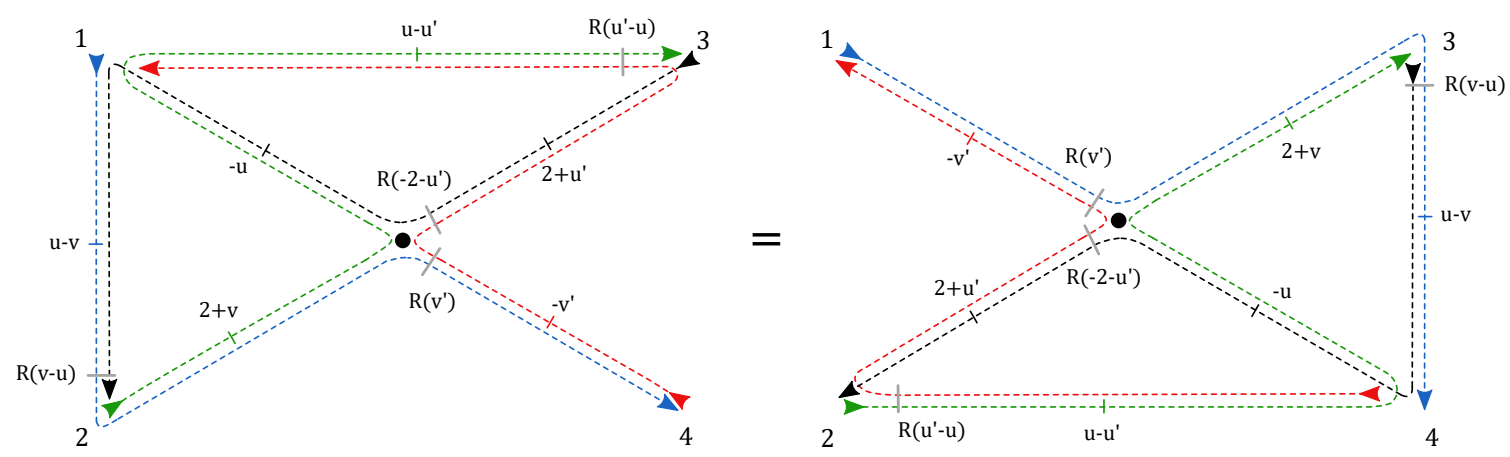

Figure 10. Diagrammatic form of the interchange relation II.

The proof of the identity consists of four star-triangle steps

- The black/blue line 12 in the l.h.s. of figure 9 is the edge of a triangle of propagators whose scaling dimensions sum to 2 , thus it can be rewritten as a star integral of vertices $\{1,2, \bullet\}$ as in figure 7 .

- The point of $\bullet$ is now the integrated point of a star integral of propagators whose scaling dimensions sum to 4 . Therefore it can be integrated delivering a triangle whose basis is a black/blue line 34, again as in figure 7. The move of the line 12 to 34 is completed.

- The same two steps can be performed on the red/green line 13, which in turn gets moved downstairs to 24, resulting in the r.h.s. of figure 9 .

The second identity differs from the first only in the mutual orientation of spinorial lines and position of $\mathbf{R}(u)$-matrices. The proof follows the very same steps as for the first relation, adapted only in the variant of star-triangle identity involved, and its diagrammatic form is: The statement of the interchange relations can be read out of figure 9 and figure 10: moving the horizontal (red/green) propagator and the vertical (grey/black) propagator across the quartic scale invariant vertex, the powers $\left(u, v, u^{\prime}, v^{\prime}\right)$ in the vertex propagators get interchanged, and so do the spinorial structures depicted by different colors of lines.

\section{General $\mathrm{SO}(1,5)$ R-operator}

In this section we present a solution of the Yang-Baxter equation for the group $\mathrm{SO}(1,5)$ in the unitary irreps of the principal series. As the representation is infinite-dimensional, the solution is an infinite dimensional $R$-operator. Its derivation is based on the startriangle duality, and we realize it in the diagrammatic notation of Feynman integrals. The knowledge of an $R$-operator is the main object needed to formulate the quantum integrability of a system of interacting particles according to the QISM approach [30,31], and it will be the topic of the next sections.

An $R$-operator $\mathcal{R}_{i j}(u)$ is an endomorphism over the tensor product of two representation modules $\mathbb{V}_{i} \otimes \mathbb{V}_{j}$ that depends on the spectral parameter $u \in \mathbb{C}$, and solves the 
Yang-Baxter equation (YBE) [32]

$$
\mathcal{R}_{12}(u-v) \mathcal{R}_{13}(u) \mathcal{R}_{23}(v)=\mathcal{R}_{23}(v) \mathcal{R}_{13}(u) \mathcal{R}_{12}(u-v),
$$

where the action of $\mathcal{R}_{i j}(u)$ on any module $\mathbb{V}_{k}$, where $k \neq i, j$ is trivial. The module $\mathbb{V}_{k}$ is the Hilbert space of the $k$-th particle of the system, i.e. the space of functions of the point $x^{\mu} \in \mathbb{R}^{4}$ with symmetric spinor indices of dotted/undotted type

$$
\Phi_{\mathbf{a} \dot{\mathbf{a}}}(x) \equiv \Phi_{\left(a_{1}, \ldots, a_{\ell}\right)\left(\dot{a}_{1}, \ldots, \dot{a}_{\ell}\right)}(x),
$$

and it carries an infinite-dimensional irrep of $\mathrm{SO}(1,5)$ labelled by the scaling dimension $\Delta \in \mathbb{C}$ and the left/right spins $(\ell, \dot{\ell})$

$$
\mathbb{V}_{k} \longleftrightarrow\left(\Delta_{k}, \ell_{k}, \dot{\ell}_{k}\right)
$$

Explicitly, the action of the $R$-operator reads

$$
\Phi\left(x_{1}\right)_{\mathbf{a} \dot{\mathbf{a}}} \Phi\left(x_{2}\right)_{\mathbf{b} \dot{\mathbf{b}}} \mapsto \Phi^{\prime}\left(u ; x_{1}, x_{2}\right)_{\mathbf{a} \mathbf{a} \mathbf{b} \dot{\mathbf{b}}}=\left(\mathcal{R}_{12}(u)\right)_{\mathbf{a} \mathbf{a} \mathbf{b} \dot{\mathbf{b}}}^{\mathbf{c} \dot{d}} \Phi\left(x_{1}\right)_{\mathbf{c} \dot{\mathbf{c}}} \Phi\left(x_{2}\right)_{\mathbf{d} \dot{\mathbf{d}}} .
$$

The function $\Phi^{\prime}\left(u, x_{1}, x_{2}\right)$ must belong to the tensor product of representations $\mathbb{V}_{1} \otimes \mathbb{V}_{2}$, implying that a solution of YBE must be conformal invariant

$$
\left(T_{1} \otimes T_{2}\right) \mathcal{R}_{12}(u)=\mathcal{R}_{12}(u)\left(T_{1} \otimes T_{2}\right),
$$

for $T_{k}$ representative of a $\mathrm{SO}(1,5)$ group element in the representation $\left(\Delta_{k}, \ell_{k}, \dot{\ell}_{k}\right)$.

A solution $\mathcal{R}_{i j}(u)$ of the YBE can be built starting from two observations [3]. First, it is always possible to formulate the problem in an equivalent way as a braid equation

$$
\hat{\mathcal{R}}_{12}(u) \hat{\mathcal{R}}_{23}(u+v) \hat{\mathcal{R}}_{12}(v)=\hat{\mathcal{R}}_{23}(v) \hat{\mathcal{R}}_{12}(u+v) \hat{\mathcal{R}}_{23}(u),
$$

where $\hat{\mathcal{R}}_{i j}(u)=\mathbb{P}_{i j} \mathcal{R}_{i j}(u)$ and $\mathbb{P}_{i j}\left(\mathbb{V}_{i} \otimes \mathbb{V}_{j}\right)=\mathbb{V}_{j} \otimes \mathbb{V}_{i}$. Secondly, the star-triangle duality (2.28) can be considered as a braid relation itself, involving operators that act on the spaces of two particles $\mathbb{V}_{1} \otimes \mathbb{V}_{2}$

$$
\begin{aligned}
S_{\ell_{k} \dot{\ell}_{k}}\left(u, \hat{p}_{k}\right) & =\hat{p}_{k}^{2 u}\left[\overline{\mathbf{p}}_{k}\right]^{\ell_{k}} \mathbf{R}_{\ell_{k} \dot{\ell}_{k}}(u)\left[\mathbf{p}_{k}\right]^{\dot{\ell}_{k}}, \\
S_{\ell_{1} \dot{\ell}_{2}}\left(u, x_{12}\right) & =x_{12}^{2 u}\left[\overline{\mathbf{x}}_{12}\right]^{\ell_{1}} \mathbf{R}_{\ell_{1} \dot{\ell}_{2}}(u)\left[\mathbf{x}_{12}\right]^{\dot{\ell}_{2}} .
\end{aligned}
$$

Hence, it is natural to look for a solution of (3.6) as a combination $\hat{\mathcal{R}}_{12}(u)$ of the operators $S\left(u, x_{12}\right)$ and $S\left(u, p_{k}\right)$ that inherits the braid property from the star-triangle duality and exchanges the representations $\left(\Delta_{1}, \ell_{1}, \dot{\ell}_{1}\right)$ and $\left(\Delta_{2}, \ell_{2}, \dot{\ell}_{2}\right)$ of the modules $\mathbb{V}_{1} \otimes \mathbb{V}_{2}$

$$
\left(T_{2} \otimes T_{1}\right) \hat{\mathcal{R}}_{12}=\hat{\mathcal{R}}_{12}\left(T_{1} \otimes T_{2}\right),
$$

as it follows from (3.5) and from the definition of $\hat{\mathcal{R}}_{12}$. Such a combination exists and reads

$$
\hat{\mathcal{R}}_{12}(u)=S_{\dot{\ell}_{1} \ell_{2}}\left(u-\Delta_{+}, x_{12}\right) S_{\ell_{2} \ell_{1}}\left(u+\Delta_{-}, \hat{p}_{2}\right) S_{\dot{\ell}_{2} \dot{\ell}_{1}}\left(u-\Delta_{-}, \hat{p}_{1}\right) S_{\ell_{1} \dot{\ell}_{2}}\left(u+\Delta_{+}, x_{12}\right),
$$


or in explicit form

$$
\begin{aligned}
\hat{\mathcal{R}}_{12}(u)= & x_{12}^{2\left(u-\Delta_{+}\right)}\left[\overline{\mathbf{x}}_{12}\right]^{\dot{\ell}_{1}} \mathbf{R}_{\dot{\ell}_{1} \ell_{2}}\left(u-\Delta_{+}\right)\left[\mathbf{x}_{12}\right]^{\ell_{2}} \hat{p}_{2}^{2\left(u+\Delta_{-}\right)}\left[\overline{\mathbf{p}}_{2}\right]^{\ell_{2}} \mathbf{R}_{\ell_{2} \ell_{1}}\left(u+\Delta_{-}\right)\left[\mathbf{p}_{2}\right]^{\ell_{1}} \\
& \times \hat{p}_{1}^{2\left(u-\Delta_{-}\right)}\left[\overline{\mathbf{p}}_{1}\right]^{\dot{\ell}_{2}} \mathbf{R}_{\dot{\ell}_{2} \dot{\ell}_{1}}\left(u-\Delta_{-}\right)\left[\mathbf{p}_{1}\right]^{\dot{\ell}_{1}} x_{12}^{2\left(u+\Delta_{+}\right)}\left[\overline{\mathbf{x}}_{12}\right]^{\ell_{1}} \mathbf{R}_{\ell_{1} \dot{\ell}_{2}}\left(u+\Delta_{+}\right)\left[\mathbf{x}_{12}\right]^{\dot{\ell}_{2}},
\end{aligned}
$$

where $\Delta_{-}=\frac{\Delta_{1}-\Delta_{2}}{2}$ and $\Delta_{+}=\frac{\Delta_{1}+\Delta_{2}}{2}-2$. The combination (3.10) is the straightforward generalization, in full analogy to (2.28) and (2.10), of the spinless solution [3]

$$
\hat{\mathcal{R}}_{12}(u)=x_{12}^{2\left(u-\Delta_{+}\right)} \hat{p}_{1}^{2\left(u-\Delta_{-}\right)} \hat{p}_{2}^{2\left(u+\Delta_{-}\right)} x_{12}^{2\left(u+\Delta_{+}\right)} .
$$

Let us analyze the behavior of the operators (3.7) and (3.8) under conformal transformations. These operators are not conformal invariants, nevertheless they define an intertwining of principal series representations, namely they map a function on $\mathbb{V}_{1} \otimes \mathbb{V}_{2}$ to the product of modules $\mathbb{V}_{1}^{\prime} \otimes \mathbb{V}_{2}^{\prime}$, carrying a different representations of the principal series [1]. In particular, for the value $u=2-\Delta$ the operator $S_{\ell \dot{\ell}}(2-\Delta, \hat{p})$ defines the map (shadow transformation) $(\Delta, \ell, \dot{\ell}) \rightarrow(4-\Delta, \dot{\ell}, \ell)$, that is intertwining relation

$$
T^{\prime} S_{\ell \dot{\ell}}(2-\Delta, \hat{p})=S_{\ell \dot{\ell}}(2-\Delta, \hat{p}) T,
$$

Note that the shadow transformation is an involution, that is expressed by the compatibility relation

$$
S_{\dot{\ell} \ell}(-u, \hat{p}) S_{\dot{\ell}}(u, \hat{p})=\mathbb{1},
$$

and holds as a consequence of the $\mathbf{R}$-matrix parity (2.22).

In the case of $S_{\ell_{1} \dot{\ell}_{2}}\left(u, x_{12}\right)$ the transformation involves both modules at the same time

$$
\begin{aligned}
\left(\Delta_{1}, \ell_{1}, \dot{\ell}_{1}\right) \otimes\left(\Delta_{2}, \ell_{2}, \dot{\ell}_{2}\right) & \rightarrow\left(\Delta_{1}-u, \dot{\ell}_{2}, \dot{\ell}_{1}\right) \otimes\left(\Delta_{2}-u, \ell_{2}, \ell_{1}\right), \\
\left(T_{1}^{\prime} \otimes T_{2}^{\prime}\right) S_{\ell_{1} \dot{\ell}_{2}}\left(u, x_{12}\right) & =S_{\ell_{1} \dot{\ell}_{2}}\left(u, x_{12}\right)\left(T_{1} \otimes T_{2}\right),
\end{aligned}
$$

and similarly to (3.14) the involutivity is expressed by the compatibility relation

$$
S_{\dot{\ell}_{2} \ell_{1}}\left(-u, x_{12}\right) S_{\ell_{1} \dot{\ell}_{2}}\left(u, x_{12}\right)=\mathbb{1} .
$$

Fixing $v=2-\Delta_{1}$ in (2.28), the two sides of the star-triangle duality

$$
\begin{aligned}
S_{\dot{\ell}_{2} \ell_{1}}\left(u, \hat{p}_{1}\right) S_{\dot{\ell}_{1} \dot{\ell}_{2}}(2+u- & \left.\Delta_{1}, x_{12}\right) S_{\ell_{1} \dot{\ell}_{1}}\left(2-\Delta_{1}, \hat{p}_{1}\right)= \\
& =S_{\dot{\ell}_{1} \ell_{1}}\left(2-\Delta_{1}, x_{12}\right) S_{\dot{\ell}_{2} \dot{\ell}_{1}}\left(2+u-\Delta_{1}, \hat{p}_{1}\right) S_{\ell_{1} \dot{\ell}_{2}}\left(u, x_{12}\right),
\end{aligned}
$$

can be regarded as two equivalent ways to compose operators (3.7) and (3.8) to realize the map between representations:

$$
\left(\Delta_{1}, \ell_{1}, \dot{\ell}_{1}\right) \otimes\left(\Delta_{2}, \ell_{2}, \dot{\ell}_{2}\right) \longrightarrow\left(u+2, \ell_{1}, \dot{\ell}_{2}\right) \otimes\left(\Delta_{1}+\Delta_{2}-u-2, \ell_{2}, \dot{\ell}_{1}\right) .
$$

In this respect the duality (3.17) together with the properties (3.14) and (3.16) are the Coxeter relations for the intertwiners of irreps of $\mathrm{SO}(1,5)$ (for a detailed discussion see [3]). 

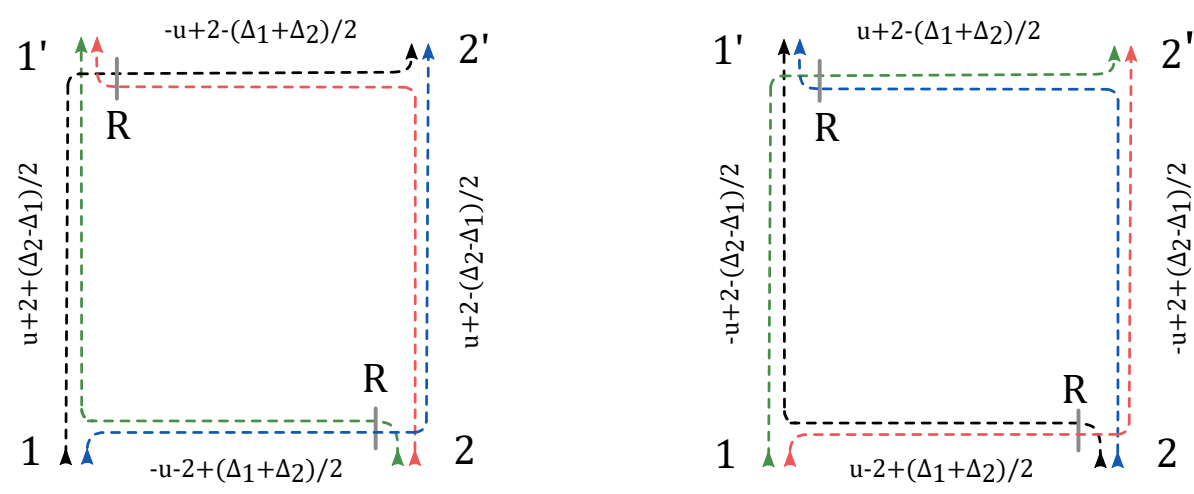

Figure 11. Left: diagram of the kernel $\hat{\mathcal{R}}_{12}\left(x_{1}, x_{2} \mid x_{1}^{\prime}, x_{2}^{\prime}\right)$ of the operator $\hat{\mathcal{R}}_{12}(u)$. The superscripts of each segment are the powers of the inverse square distance between the end points. Grey solid lines represent the fused $\mathrm{SU}(2) \mathrm{R}$-matrix acting along the spinorial structure of the propagators. The argument of matrices $\mathbf{R}$ are the opposite of the power of the adjancent line. Right: diagram of the kernel of the operator $\hat{\mathcal{R}}_{12}^{-1}(u)$.

According to the definition (3.10), the solution of the braid relation $\hat{\mathcal{R}}_{12}$ acts on functions of the space coordinates $x_{1}$ and $x_{2}$ as an integral operator

$$
\hat{\mathcal{R}}_{12}(u) \Phi\left(x_{1}, x_{2}\right)=\frac{1}{a_{\dot{\ell}_{2} \dot{\ell}_{1}}\left(u-\Delta_{-}\right) a_{\ell_{2} \ell_{1}}\left(u+\Delta_{-}\right)} \int d^{4} x_{1}^{\prime} d^{4} x_{2}^{\prime} \hat{\mathcal{R}}\left(x_{1}, x_{2} \mid x_{1}^{\prime}, x_{2}^{\prime}\right) \Phi\left(x_{1}^{\prime}, x_{2}^{\prime}\right) .
$$

Its kernel $\hat{\mathcal{R}}\left(x_{1}, x_{2} \mid x_{1}^{\prime}, x_{2}^{\prime}\right)$ in compact notation reads

$$
\frac{\left[\overline{\mathbf{x}}_{12}\right]^{\dot{\ell}_{1}} \mathbf{R}_{\dot{\ell}_{1} \ell_{2}}\left(u-\Delta_{+}\right)\left[\mathbf{x}_{12}\right]^{\ell_{2}}}{x_{12}^{2\left(-u+\Delta_{+}\right)}} \frac{\left[\overline{\mathbf{x}}_{22^{\prime}}\right]^{\ell_{2}}\left[\mathbf{x}_{22^{\prime}}\right]^{\ell_{1}}\left[\overline{\mathbf{x}}_{11^{\prime}}\right]^{\dot{\ell}_{2}}\left[\mathbf{x}_{11^{\prime}}\right]^{\dot{\ell}_{1}}}{x_{22^{\prime}}^{2\left(u+\Delta_{-}+2\right)}} \frac{\left[\overline{\mathbf{x}}_{1^{\prime} 2^{\prime}}\right]^{\ell_{1}} \mathbf{R}_{\ell_{1^{\prime}} \dot{\ell}_{2}}\left(u+\Delta_{+}\right)\left[\mathbf{x}_{1^{\prime} 2^{\prime}}\right]^{\dot{\ell}_{2}}}{x_{11^{\prime}}^{2\left(u-\Delta_{-}+2\right)}},
$$

and as a diagram it is a square of propagators with spinor mixing, as depicted in figure 11. Using (3.14) and (3.16) it is easy to construct inverse operator $\hat{\mathcal{R}}_{12}^{-1}(u)$ and its kernel is shown in the same figure 11.

The operator $\mathcal{R}_{12}(u)$ enjoys a simple behavior under hermitean conjugation; the form of the YBE ensures that $\mathcal{R}_{12}^{\dagger}(u)$ is still a solution. For the $S$-operator we have

$$
S_{\ell \dot{\ell}}^{\dagger}(u, \hat{p})=\hat{p}^{2 u^{*}}[\overline{\mathbf{p}}]^{\dot{\ell}} \mathbf{R}_{\ell \dot{\ell}}\left(u^{*}\right)[\mathbf{p}]^{\ell}=S_{\dot{\ell} \ell}\left(u^{*}, \hat{p}\right)=S_{\dot{\ell}}^{-1}\left(-u^{*}, \hat{p}\right),
$$

and the same in coordinate representation. Then from the definition (3.10) it follows that

$$
\mathcal{R}_{12}^{\dagger}\left(u, \Delta_{ \pm}\right)=\mathcal{R}_{12}^{-1}\left(-u^{*},-\Delta_{ \pm}^{*}\right) .
$$

where we show explicitly dependence on the parameters $\Delta_{ \pm}$in $\mathcal{R}$-operator. For the unitary series representations of conformal group parameters are fixed as follows: $\Delta_{1}=$ $2+i \nu_{1}, \Delta_{2}=2+i \nu_{2}$ so that $\Delta_{-}=\frac{i}{2}\left(\nu_{1}-\nu_{2}\right)$ and $\Delta_{+}=\frac{i}{2}\left(\nu_{1}+\nu_{2}\right)$ are pure imaginary; in this case for imaginary spectral parameter $\mathcal{R}_{12}(u)$ is a unitary operator. The integral kernels of the operators $\mathcal{R}_{12}(u), \mathcal{R}_{12}^{\dagger}(u)$ are drawn in figure 12.

Since the Yang-Baxter equation for $\mathcal{R}_{i j}(u)$ is ultimately a consequence of the braid relations satisfied by the operators (3.7) and (3.8), its proof can delivered in the diagrammatic formalism developed in section 2, with few transparent steps. For a choice of three 

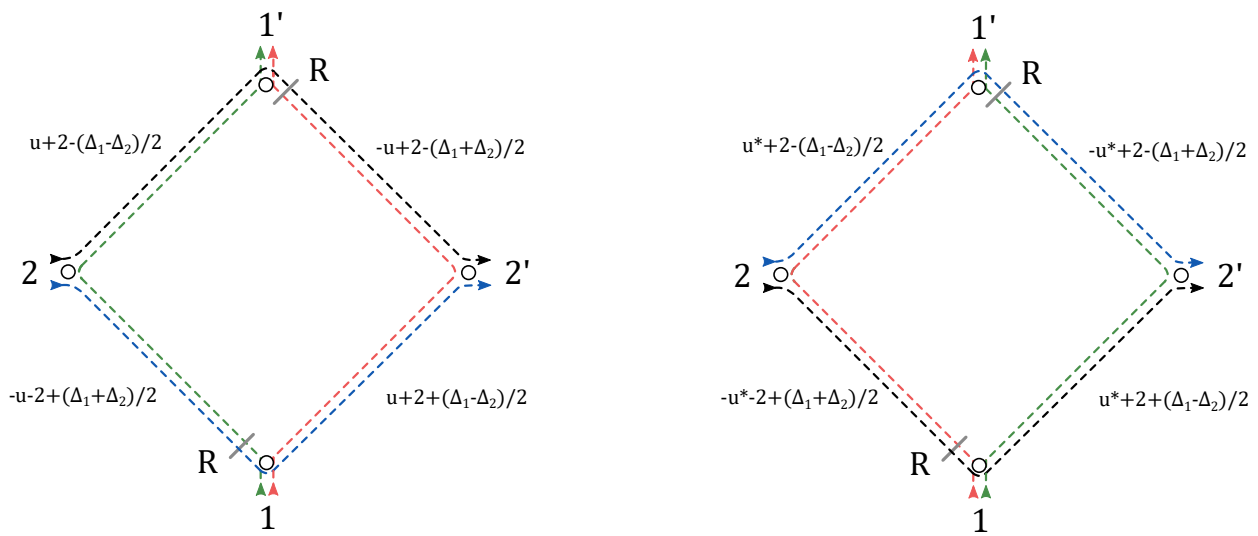

Figure 12. Left: diagrammatic representation of the kernel $\mathcal{R}_{12}\left(x_{1}, x_{2} \mid x_{1}^{\prime}, x_{2}^{\prime}\right)$ of the operator $\mathcal{R}_{12}(u)$. It is obtained from the kernel of figure 11 (left) by exchange of the points 1 and 2 following from the definition $\mathcal{R}_{12}=\mathbb{P}_{12} \hat{\mathcal{R}}_{12}$. Right: diagrammatic representation of the kernel of the operator $\mathcal{R}_{12}^{\dagger}(u)$. The color of lines - that is the values of the spins - get exchanged by the hermitean conjugation.

particles/modules in any unitary irreps

$$
\mathbb{V}_{1} \rightarrow\left(\Delta_{1}, \ell_{1}, \dot{\ell}_{1}\right), \quad \mathbb{V}_{2} \rightarrow\left(\Delta_{2}, \ell_{2}, \dot{\ell}_{2}\right), \quad \mathbb{V}_{3} \rightarrow\left(\Delta_{3}, \ell_{3}, \dot{\ell}_{3}\right)
$$

the l.h.s. of 12 is a convolution of the integral kernels of $\mathcal{R}_{i j}$ operators (left picture). Here each color denotes spinorial indices - carried by matrices $\sigma, \bar{\sigma}$ - belonging to different spinor spaces. The first step in the proof consist in redrawing the triangle $\left(y_{1}, y_{2}, y_{3}\right)$ as a star integral via the identity (2.31) (right picture)
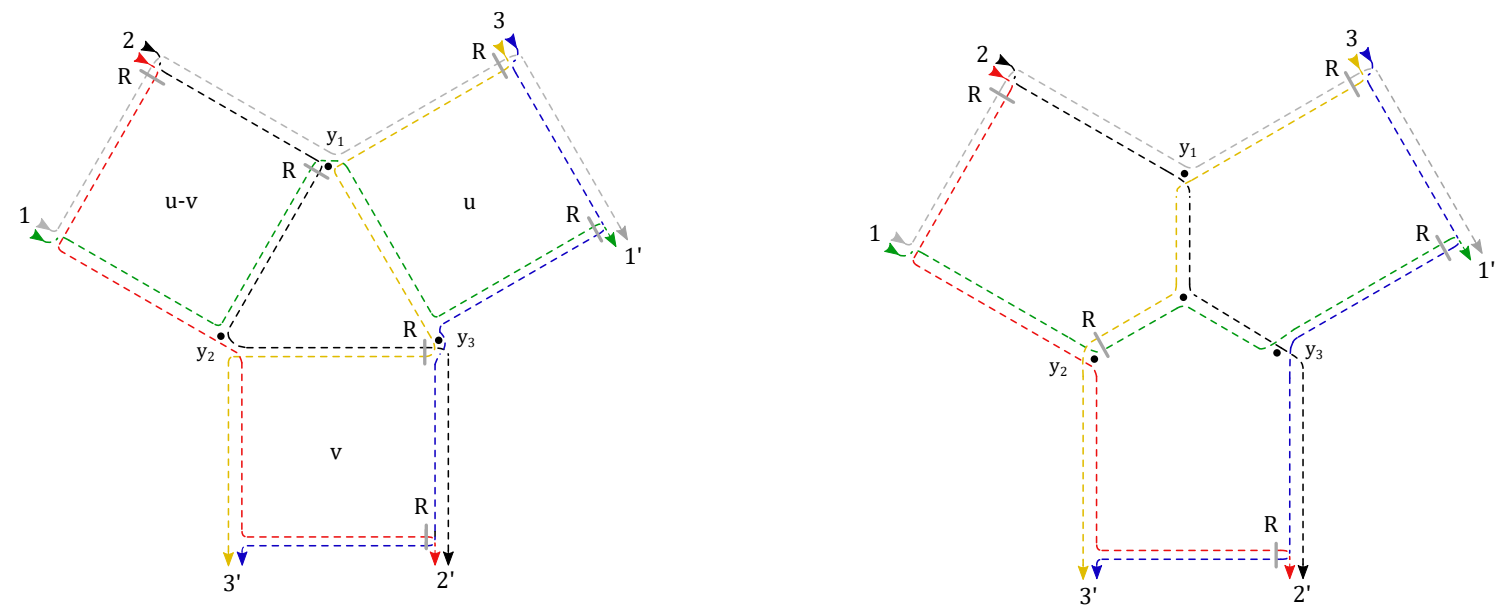

At the second step, we apply the same identity (8) to integrate the scale invariant vertices $y_{1}, y_{2}$ and $y_{3}$, transforming each star into a triangle, and obtaining the symmetric picture: 

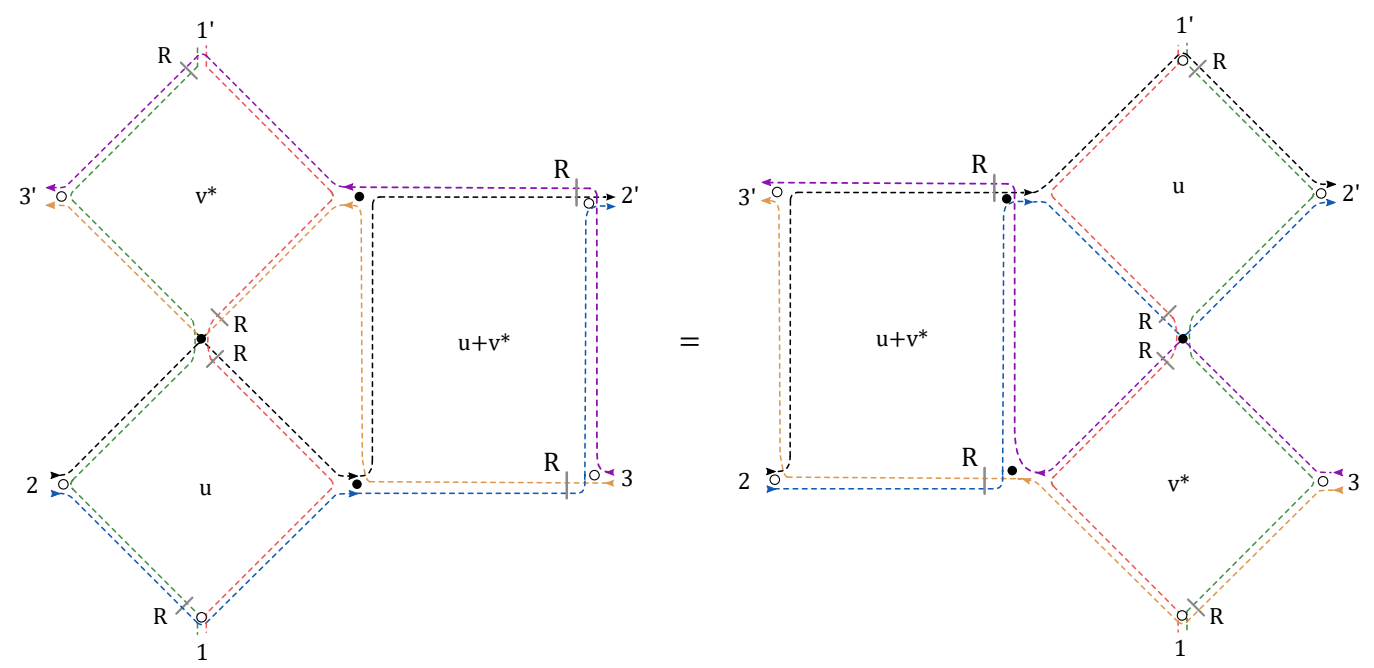

Figure 13. Diagrammatic representation of the equation (3.21). Different colors refer to different spinorial structures, black blobs indicate corresponding integrations and circles indicate external coordinates without any integration. Letters $u, v, u+v^{*}$ refer to the spectral parameters carried by the R-operators involved.

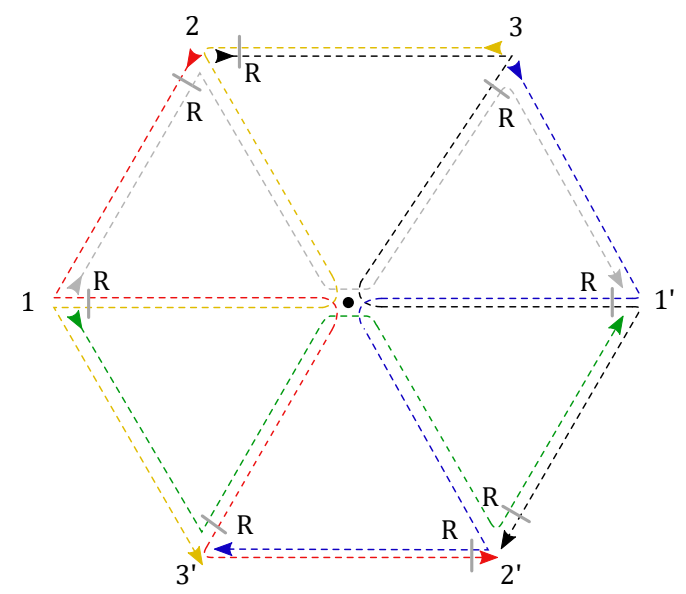

The same two steps can now be repeated starting from the r.h.s. of the (12), which amounts to a different order in the convolution of kernels $\mathcal{R}$. It is straightforward to check that as a final step one obtains the same sextic vertex of propagators, proving the Yang-Baxter property

The diagrammatic technique applied to the proof of the YBE can be repeated stepby-step to prove the relation $\mathcal{R} \mathcal{R} \mathcal{R}^{\dagger}=\mathcal{R}^{\dagger} \mathcal{R} \mathcal{R}$ (3.21) represented in figure 13. This kind of graphical computations are ubiquitous in our paper as they heavily simplify tedious analytic computations. In a similar way as the YBE, it is possible to prove that $\mathcal{R}$ and $\mathcal{R}^{\dagger}$ satisfy the simple algebra (see diagrammatic representation in figure 13)

$$
\mathcal{R}_{12}(u) \mathcal{R}_{32}\left(u+v^{*}\right) \mathcal{R}_{13}(v)^{\dagger}=\mathcal{R}_{13}(v)^{\dagger} \mathcal{R}_{32}\left(u+v^{*}\right) \mathcal{R}_{12}(u) .
$$



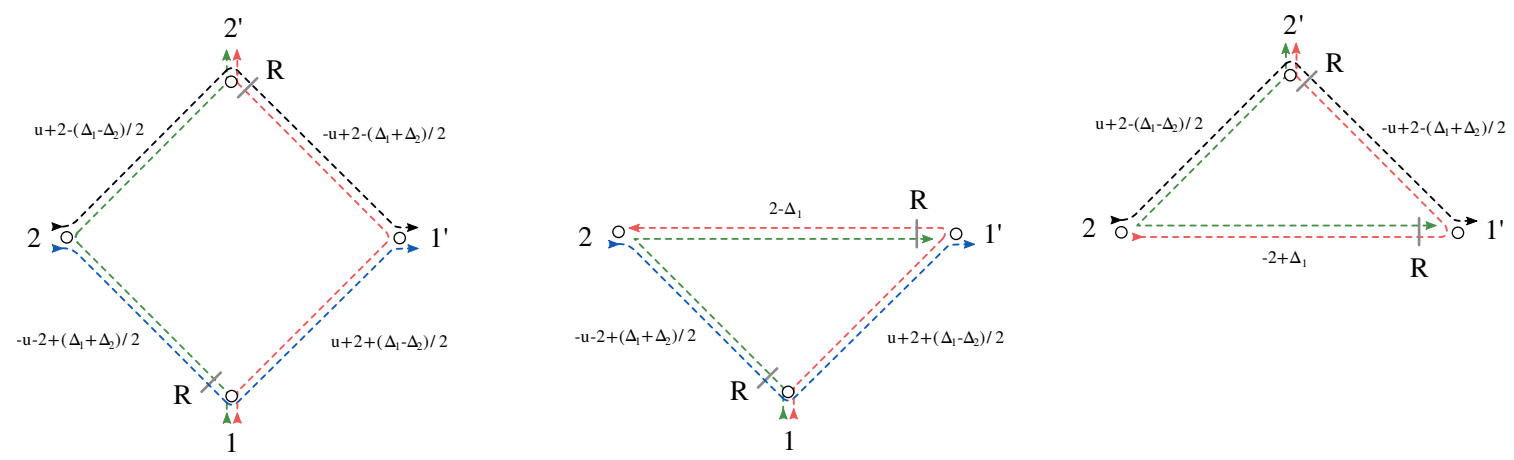

Figure 14. Left: the kernel of the general $\hat{\mathcal{R}}$-operator in diagram formalism. It can be factorized into the product of two integral kernels $\hat{\mathcal{R}}_{ \pm}$. Center: integral kernel of the operator $\hat{\mathcal{R}}_{-}(u)$ Right: integral kernel of the operator $\hat{\mathcal{R}}_{+}(u)$. When multiplied, the horizontal red/green lines in the two triangular kernels simplify, as their power is opposite as well as the argument of the two R-matrices involved (see $(2.22)$ ) and the kernel of $\hat{\mathcal{R}}(u)$ is recovered.

We introduce here for later purposes a factorization of the operator $\hat{\mathcal{R}}(u)$ as the product of two operators $\hat{\mathcal{R}}_{12}(u)=\hat{\mathcal{R}}_{12}^{-}(u) \hat{\mathcal{R}}_{12}^{+}(u)$, where

$$
\begin{aligned}
& \hat{\mathcal{R}}_{12}^{+}(u)=S_{\dot{\ell}_{1} \ell_{1}}\left(2-\Delta_{1}, x_{12}\right) S_{\dot{\ell}_{2} \dot{\ell}_{1}}\left(u-\Delta_{-}, \hat{p}_{1}\right) S_{\ell_{1} \dot{\ell}_{2}}\left(u+\Delta_{+}, x_{12}\right)= \\
& =\frac{\left[\overline{\mathbf{x}}_{12}\right]^{\dot{\ell}_{1}} \mathbf{R}_{\dot{\ell}_{1} \ell_{1}}\left(2-\Delta_{1}\right)\left[\mathbf{x}_{12}\right]^{\ell_{1}}}{x_{12}^{2\left(\Delta_{1}-2\right)}} \frac{\left[\overline{\mathbf{p}}_{1}\right]^{\dot{\ell}_{2}} \mathbf{R}_{\dot{\ell}_{2} \dot{\ell}_{1}}\left(u-\Delta_{-}\right)\left[\mathbf{p}_{1}\right]^{\dot{\ell}_{1}}}{\hat{p}_{1}^{2\left(\Delta_{-}-u\right)}} \frac{\left[\overline{\mathbf{x}}_{12}\right]^{\ell_{1}} \mathbf{R}_{\ell_{1} \dot{\ell}_{2}}\left(u+\Delta_{+}\right)\left[\mathbf{x}_{12}\right]^{\dot{\ell}_{2}}}{x_{12}^{-2\left(u+\Delta_{+}\right)}}
\end{aligned}
$$

and

$$
\begin{aligned}
& \hat{\mathcal{R}}_{12}^{-}(u)=S_{\dot{\ell}_{1} \ell_{2}}\left(u-\Delta_{+}, x_{12}\right) S_{\ell_{2} \ell_{1}}\left(u+\Delta_{-}, \hat{p}_{2}\right) S_{\ell_{1} \dot{\ell}_{1}}\left(\Delta_{1}-2, x_{12}\right)= \\
& =\frac{\left[\overline{\mathbf{x}}_{12}\right]^{\dot{\ell}_{1}} \mathbf{R}_{\dot{\ell}_{1} \ell_{2}}\left(u-\Delta_{+}\right)\left[\mathbf{x}_{12}\right]^{\ell_{2}}}{x_{12}^{2\left(\Delta_{+}-u\right)}} \frac{\left[\overline{\mathbf{p}}_{2}\right]^{\ell_{2}} \mathbf{R}_{\ell_{2} \ell_{1}}\left(u+\Delta_{-}\right)\left[\mathbf{p}_{2}\right]^{\ell_{1}}}{\hat{p}_{2}^{-2\left(u+\Delta_{-}\right)}} \frac{\left[\overline{\mathbf{x}}_{12}\right]^{\ell_{1}} \mathbf{R}_{\ell_{1} \dot{\ell}_{1}}\left(\Delta_{1}-2\right)\left[\mathbf{x}_{12}\right]^{\dot{\ell}_{1}}}{x_{12}^{2\left(2-\Delta_{1}\right)}} .
\end{aligned}
$$

\section{Inhomogeneous spinning Fishnet}

The existence of an $\mathcal{R}$-operator acting on any two unitary irreps of the conformal group $\mathrm{SO}(1,5)$ allows to define a family of integrable spin chains with conformal symmetry. These systems are the generalization of the $\mathrm{SU}(2)$ Heisenberg magnet $[30,31]$ to the conformal group $\mathrm{SO}(1,5)$, being a chain of nearest-neighbour interacting sites. Each site carries a vector in the module of a given unitary irrep of the conformal group [14] — that is the wave function of a conformal particle in the Euclidean $4 d$ space. The states of the spin chain with $L$ sites belong to the Hilbert space

$$
\mathcal{V}=\mathbb{V}_{1} \otimes \mathbb{V}_{2} \otimes \cdots \otimes \mathbb{V}_{L}
$$

where each $\mathbb{V}_{k}$ is the module of the representation $\left(\Delta_{k}, \ell_{k}, \dot{\ell}_{k}\right)$ - in general different at each site - and the scalar product on $\mathcal{V}$ is inherited by those on the sites $\mathbb{V}_{k}$ defined 


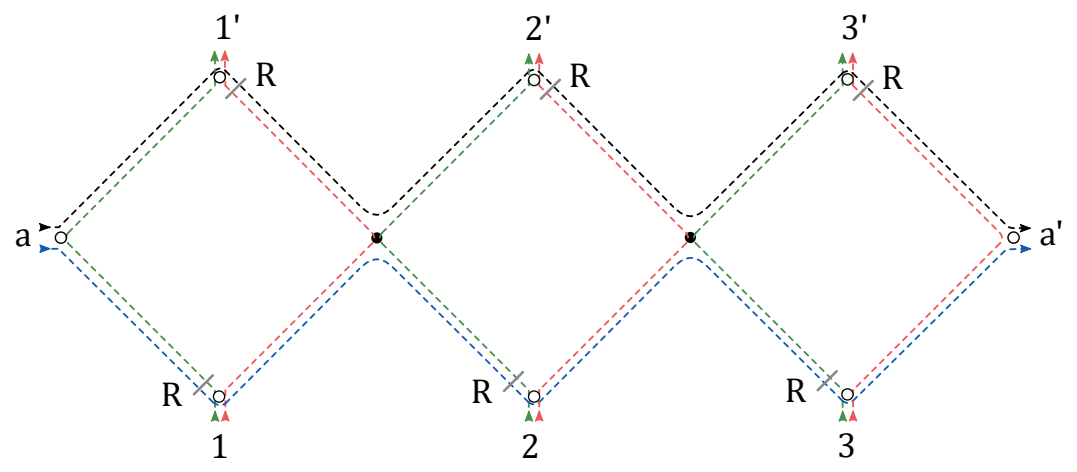

Figure 15. Integral kernel of the monodromy matrix operator $\mathbb{T}_{1,2,3, a}(u)$ obtained from the convolution of operators $\mathcal{R}_{k a}\left(u+\theta_{k}\right)$ in the auxiliary space $\mathbb{V}_{a}$ for $k=1,2,3$. The black blobs are integrated, the circles are external coordinates. Black and blue lines denote the propagation of Weyl spinors in the auxiliary space $\left(\ell_{a}, 0\right)$ and $\left(0, \dot{\ell}_{a}\right)$, while the red and green lines are Weyl spinor propagators in the representations $\left(\ell_{k}, 0\right)$ or $\left(0, \dot{\ell}_{k}\right)$ of the physical spaces $\mathbb{V}_{k}, k=1, \ldots, L$. The mixing of spinorial indices between auxiliary (black, blue) and physical (red, green) representations is given by the $\mathrm{SU}(2)$ R-matrices denoted with a grey line. The transfer matrix $t^{(a)}(u)$ is obtained by the identification and integration of the points $x_{a}$ and $x_{a}^{\prime}$.

in (2.3). The wave function of the system depends on the position of the particles $x_{k}^{\mu} \in \mathbb{R}$ and carries $\ell_{k^{-}}$and $\dot{\ell}_{k}$-symmetric spinor indices

$$
\Phi_{\mathbf{a}_{1} \dot{\mathbf{a}}_{1} \ldots \mathbf{a}_{\mathbf{L}} \dot{\mathbf{a}}_{\mathbf{L}}}\left(x_{1}, \ldots, x_{L}\right), \quad \mathbf{a}_{\mathbf{k}}=\left(a_{k, 1}, \ldots, a_{k, \ell_{k}}\right), \dot{\mathbf{a}}_{\mathbf{k}}=\left(\dot{a}_{k, 1}, \ldots, \dot{a}_{k, \dot{\ell}_{k}}\right) .
$$

Under the action of the conformal group these functions transform in the tensor product of the representations defined at each site. We consider a closed spin chain, where the $(L+1)$-th particle is identified with the 1-st one, with periodic boundary conditions. ${ }^{2}$ The dynamics of the system can be introduced starting from the definition of a monodromy matrix operator

$$
\mathbb{T}_{1, \ldots, L, a}(u)=\mathcal{R}_{1 a}\left(u+\theta_{1}\right) \mathcal{R}_{2 a}\left(u+\theta_{2}\right) \cdots \mathcal{R}_{L a}\left(u+\theta_{L}\right),
$$

which acts on the physical space of system $\mathcal{V}$ and on an auxiliary space $\mathbb{V}_{a}$, chosen to be the module of the unitary irrep $\left(\Delta_{a}, \ell_{a}, \dot{\ell}_{a}\right)$.

As a consequence of the YBE (3.1) the monodromy operator satisfies the RTT relation (Yangian algebra)

$$
\mathcal{R}_{a b}(u-v) \mathbb{T}_{1, \ldots, L, a}(u) \mathbb{T}_{1, \ldots, L, b}(v)=\mathbb{T}_{1, \ldots, L, a}(u) \mathbb{T}_{1, \ldots, L, b}(v) \mathcal{R}_{a b}(u-v) .
$$

The algebra (4.4) allows to define an infinite family of commuting transfer matrix operators

$$
t^{(a)}(u) t^{(b)}(v)=t^{(b)}(v) t^{(a)}(u),
$$

as the infinite dimensional trace in the auxiliary spaces $\mathbb{V}_{a} \otimes \mathbb{V}_{b}$ of monodromies

$$
t^{(a)}(u) \equiv t_{1, \ldots, L}^{(a)}(u) \equiv \operatorname{Tr}_{\mathbb{V}_{a}}\left(\mathbb{T}_{1, \ldots, L, a}(u)\right),
$$

\footnotetext{
${ }^{2}$ This condition can be relaxed by the introduction of a twist at the boundary while preserving the integrability of the model.
} 
Each transfer matrix depends through the label $a$ on one of the infinite possible principal series irreps chosen for the auxiliary space. As analytic functions of the parameter $u \in \mathbb{R}$, the operators $t^{(a)}(u)$ can be Taylor expanded around a chosen $u=u_{0}$ leading to an infinite tower of commuting operators

$$
H_{k}^{(a)}=\left[\frac{1}{k !} \frac{d^{k}}{d u^{k}} t^{(a)}(u)\right]_{u=u_{0}}, \quad H_{i}^{(a)} H_{j}^{(b)}=H_{j}^{(b)} H_{i}^{(a)} .
$$

The definition of an integrable model with commuting charges $H^{(a)}$ requires that such operators are diagonalizable. In other words, we should require the operator $t^{(a)}(u)$ to be normal, i.e. to commute with its hermitean conjugate

$$
\left[t^{(a)}(u), t^{(a)}(u)^{\dagger}\right]=0 .
$$

The previous equation is actually a particular case of the general property

$$
t^{(a)}(u)\left(t^{(b)}(v)\right)^{\dagger}=\left(t^{(b)}(v)\right)^{\dagger} t^{(a)}(u), \quad \forall u, v,
$$

valid for any two auxiliary space representations $\left(\Delta_{a}, \ell_{a}, \dot{\ell}_{a}\right)$ and $\left(\Delta_{b}, \ell_{b}, \dot{\ell}_{b}\right)$ under the constraint

$$
\theta_{k}+\theta_{k}^{*}=\theta_{j}+\theta_{j}^{*}, \quad \forall j, k=1, \ldots, L .
$$

The proof of (4.9) makes use of the R-operator property (3.21), in the same way that (4.5) follows from the YBE. First, we can write the hermitean conjugate of the monodromy operator

$$
\mathbb{T}_{1, \ldots, L, a}^{\dagger}(u)=\mathcal{R}_{L a}\left(u+\theta_{L}\right)^{\dagger} \cdots \mathcal{R}_{2 a}\left(u+\theta_{2}\right)^{\dagger} \mathcal{R}_{1 a}\left(u+\theta_{1}\right)^{\dagger},
$$

where by $\dagger$ we mean hermitean conjugation in both spaces, physical and auxiliary, for each R-operator. Making use of (3.21) we can write a $T R T^{\dagger}$ relation

$$
\mathbb{T}_{1, \ldots, L, a}(u) \mathcal{R}_{b a}\left(u+v^{*}+\theta+\theta^{*}\right) \mathbb{T}_{1, \ldots, L, b}(v)^{\dagger}=\mathbb{T}_{1, \ldots, L, b}(v)^{\dagger} \mathcal{R}_{b a}\left(u+v^{*}+\theta+\theta^{*}\right) \mathbb{T}_{1, \ldots, L, a}(u),
$$

where $\theta+\theta^{*}=\theta_{k}+\theta_{k}^{*}$ for any $k=1, \ldots, L$. The algebra (4.9) follows from the trace over auxiliary spaces in the previous equation, where

$$
\left(t^{(a)}(u)\right)^{\dagger}=\operatorname{Tr}_{\mathbb{V}_{a}}\left(\mathbb{T}_{1, \ldots, L, a}^{\dagger}(u)\right)=\operatorname{Tr}_{\mathbb{V}_{a}}\left(\mathcal{R}_{L a}\left(u+\theta_{L}\right)^{\dagger} \cdots \mathcal{R}_{2 a}\left(u+\theta_{2}\right)^{\dagger} \mathcal{R}_{1 a}\left(u+\theta_{1}\right)^{\dagger}\right)
$$

Notice that the constraint (4.10) is compatible with the choice

$$
\theta_{k}=-\frac{\Delta_{k}}{2}=-1-i \nu_{k}
$$

which is particularly suitable to reduce $t^{(a)}(u)$ at special values of $u$ as explained in the next section.

Whenever (4.10) is satisfied the operators $t^{(a)}(u)$ are normal operators for any choice of auxiliary space and $u$, therefore for its Taylor coefficients it holds $\left[H_{k}^{(a)}, H_{j}^{(b) \dagger}\right]=0$. Thus 
$H_{k}^{(a)}$ are a continuous infinity of mutually diagonalizable operators. The Hamiltonian of the spin chain is usually picked to be the logarithmic derivative of the transfer matrix [33]

$$
\mathbb{H} \equiv\left(H_{0}^{(a)}\right)^{-1} H_{1}^{(a)}\left(u_{0}\right)
$$

The operator (4.15) takes the form of a sum of two-particle Hamiltonians acting on nearestneighbouring particles, prior to the sufficient condition that at $u=u_{0}$ each R-operator $\mathcal{R}_{i a}(u)$ inside the trace in $t^{(a)}(u)$ reduces to the permutation $\mathbb{P}_{i a}$. This fact is possible only for the homogeneous chain, that is when the representations of the conformal group in each site and in auxiliary space are the same $\left(\Delta_{k}, \ell_{k}, \dot{\ell}_{k}\right)=\left(\Delta_{a}, \ell_{a}, \dot{\ell}_{a}\right)=(\Delta, \ell, \dot{\ell})$, and all shifts are the same $\theta_{k}=\theta$. For convenience we set $\theta=0$, so that $u_{0}=0$. The explicit expression for the two-particle Hamiltonian can be obtained from the solution of the braid equation (3.10); in the case of homogeneous chain we have $\Delta_{-}=0, \Delta_{+}=\Delta-2$ and $\ell_{1}=\ell_{2}=\ell, \dot{\ell}_{1}=\dot{\ell}_{2}=\dot{\ell}$ so that

$\hat{\mathcal{R}}_{12}(u)=\frac{\left[\overline{\mathbf{x}}_{12}\right]^{\dot{\ell}} \mathbf{R}_{\dot{\ell} \ell}\left(u-\Delta_{+}\right)\left[\mathbf{x}_{12}\right]^{\ell}}{x_{12}^{2\left(-u+\Delta_{+}\right)}} \frac{\left[\overline{\mathbf{p}}_{2}\right]^{\ell} \mathbf{R}_{\ell \ell}(u)\left[\mathbf{p}_{2}\right]^{\ell}}{\hat{p}_{2}^{-2 u}} \frac{\left[\overline{\mathbf{p}}_{1}\right]^{\dot{\ell}} \mathbf{R}_{\dot{\ell} \dot{\ell}}(u)\left[\mathbf{p}_{1}\right]^{\dot{\ell}}}{\hat{p}_{1}^{-2 u}} \frac{\left[\overline{\mathbf{x}}_{12}\right]^{\ell} \mathbf{R}_{\ell \dot{\ell}}\left(u+\Delta_{+}\right)\left[\mathbf{x}_{12}\right]^{\dot{\ell}}}{x_{12}^{2\left(-u-\Delta_{+}\right)}}$.

The condition $\hat{\mathcal{R}}_{12}(0)=\mathbb{P}_{12} \mathcal{R}_{12}(0)=\mathbb{1}$ is satisfied and

$$
\mathbb{H}_{12}=\hat{\mathcal{R}}_{12}^{\prime}(u)=2 \ln x_{12}^{2}+S_{12}^{-1}\left(\ln \hat{p}_{1}^{2}+\ln \hat{p}_{2}^{2}+\left[\overline{\mathbf{p}}_{1}\right]^{\dot{\ell}} \mathbf{R}_{\dot{\ell} \dot{\ell}}^{\prime}(0)\left[\mathbf{p}_{1}\right]^{\dot{\ell}}+\left[\overline{\mathbf{p}}_{2}\right]^{\ell} \mathbf{R}_{\ell \ell}^{\prime}(0)\left[\mathbf{p}_{2}\right]^{\ell}\right) S_{12},
$$

where

$$
S_{12}=x_{12}^{2 \Delta_{+}}\left[\overline{\mathbf{x}}_{12}\right]^{\ell} \mathbf{R}_{\ell \dot{\ell}}\left(\Delta_{+}\right)\left[\mathbf{x}_{12}\right]^{\dot{\ell}} .
$$

Note that operators $\mathbf{R}_{\dot{\ell} \dot{\ell}}^{\prime}(0)$ and $\mathbf{R}_{\ell \ell}^{\prime}(0)$ are the standard Hamiltonians [33] of the $\mathrm{SU}(2)$ invariant spin chains acting for the symmetric representations $\dot{\ell}$ and $\ell$ respectively. In the case of zero spins $\mathbb{H}_{12}$ reduces to the $4 d$ version of Lipatov's Hamiltonian [3]

$$
\mathbb{H}_{12}=\hat{\mathcal{R}}_{12}^{\prime}(u)=2 \ln x_{12}^{2}+x_{12}^{-2 \Delta_{+}}\left(\ln \hat{p}_{1}^{2}+\ln \hat{p}_{2}^{2}\right) x_{12}^{2 \Delta_{+}},
$$

and (4.17) is its spinning generalization.

It follows from the definition of $\mathbb{H}$, that any other operator $H_{k}^{(a)}$ is a conserved charge ${ }^{3}$

$$
\left[\mathbb{H}, H_{i}^{(a)}\right]=0 \text {. }
$$

The number of degrees of freedom of the system of $L$ particles is $6 L$, since each particle in the chain has two $\mathrm{SU}(2)$ spins and four coordinates, i.e. six degrees of freedom. One can extract from the infinite tower of conserved charges $\left\{H_{k}^{(a)}\right\}$, a maximal subset of $6 L$ linearly independent operators so to establish the complete integrability of the quantum model.

An alternative, equivalent, definition of transfer matrices can be done starting from the factorization (3.22) (3.23), inherited by the transfer matrix operator

$$
t^{(a)}(u)=t_{-}^{(a)}(u) t_{+}^{(a)}(u) .
$$

The interpretation of (4.20) becomes very transparent in the diagrammatic notation of figure 16 .

\footnotetext{
${ }^{3}$ In this respect, any other $H_{k}^{(a)}$ or hermitean linear combinations thereof can be taken as the Hamiltonian of the system. This includes infinite linear combinations, for instance $H_{0}^{(a)}=t^{(a)}\left(u_{0}\right)$ itself.
} 

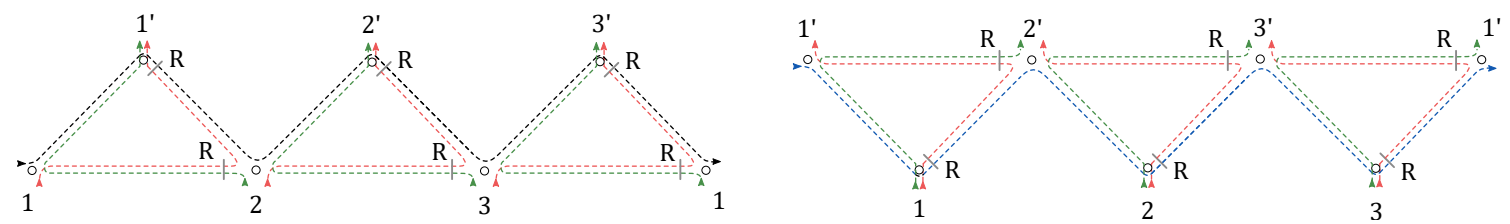

Figure 16. Left: integral kernel $t_{+}\left(x_{1}, x_{2}, x_{3} \mid x_{1}^{\prime}, x_{2}^{\prime}, x_{3}^{\prime}\right)(u)$ of the operator $t_{+}(u)$. The black dashed segments transform in the $\ell_{a}$-symmetric irrep of SU(2). Right: integral kernel $t_{-}\left(x_{1}, x_{2}, x_{3} \mid x_{1}^{\prime}, x_{2}^{\prime}, x_{3}^{\prime}\right)(u)$ of the operator $t_{-}(u)$. The blue dashed segments transform in the $\dot{\ell}_{a^{-}}$ symmetric irrep of $\mathrm{SU}(2)$.

Both operators $t_{ \pm}^{(a)}(u)$ act on the physical space $\mathcal{V}$ and carry respectively only one spin $\left(\ell_{a}, 0\right)$ or $\left(0, \dot{\ell}_{a}\right)$ in the auxiliary space, as made clear in figure 16 . By means of the interchange relations of figure 9 one can directly verify the commutation

$$
t_{+}^{(a)}(u) t_{-}^{(b)}(v)=t_{-}^{(b)}(v) t_{+}^{(a)}(u) .
$$

Indeed, starting from the l.h.s. of (4.21), depicted on the left, it is possible to insert the identity along the convolution in the auxiliary space in the form $\hat{S}_{a b}\left(u-v, x_{11^{\prime}}\right) \hat{S}_{a b}(v-$ $\left.u, x_{11^{\prime}}\right)$, as on the right picture.
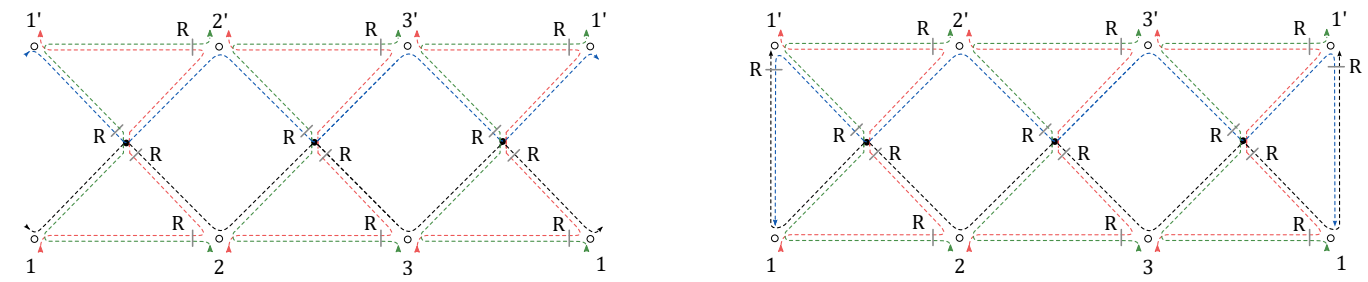

The interchange relation of figure 9 can be applied in order to move the black/blue lines $\hat{S}_{a b}\left(u-v, x_{11^{\prime}}\right)$ from the left to the right of the diagram, moving downstairs the horizontal red/green lines in the upper part of the diagram. One can easily recognize that the result amounts to the r.h.s. of (4.21).

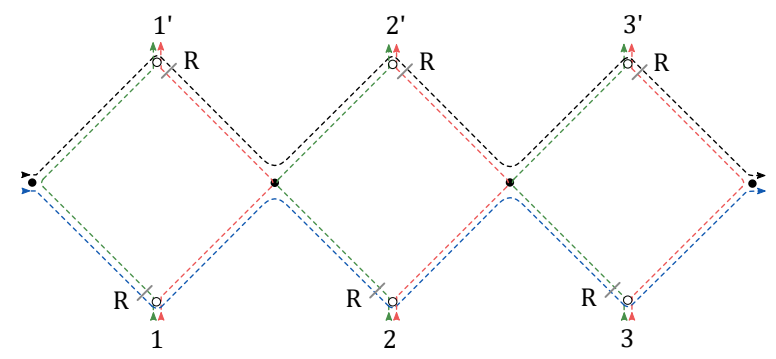

Moreover, under the constraint (4.10), the following commutation holds

$$
t_{ \pm}^{(a)}(u) t_{ \pm}^{(b)}(v)=t_{ \pm}^{(b)}(v) t_{ \pm}^{(a)}(u)
$$

The proof can be delivered via diagrams in few steps: starting from $t_{+}^{(a)}(u) t_{+}^{(b)}(v)$ - depicted on the left - the triangles corresponding to $\mathcal{R}^{+}(v)$ get opened into star integrals by means of (8), obtaining the picture on the right: 

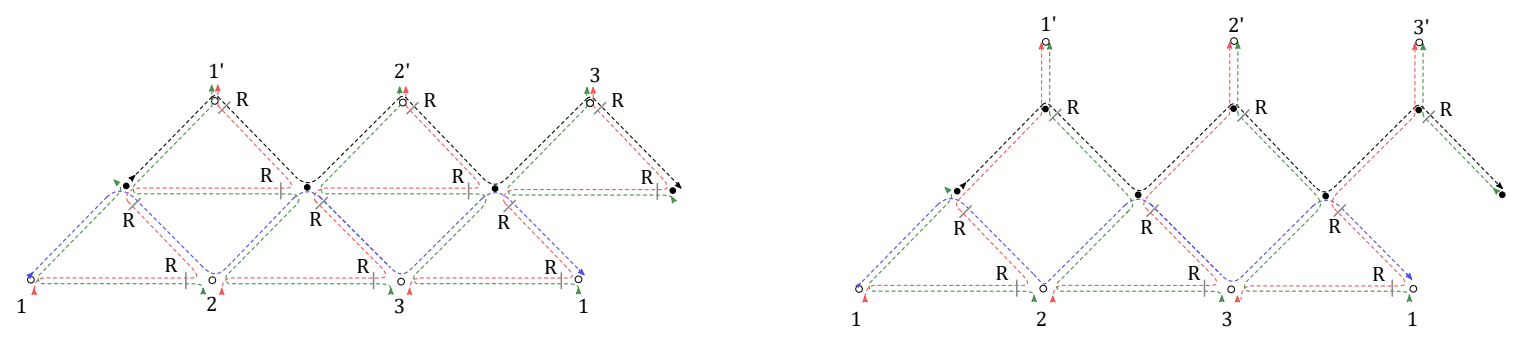

The equation (4.22) amounts to the exchange of auxiliary spaces $\mathbb{V}_{a}$ and $\mathbb{V}_{b}$ and the spectral parameters. In order to do so we introduce along the auxiliary spaces (blue/black dashed lines) the identity in the form $S_{a b}(u-v) S_{a b}(v-u)=\mathbb{1}$, that is two couples of black/blue vertical lines, as in the left picture. Now, by means of the interchange relation of figure 9 one can move the lines on the left, namely $S_{a b}(u-v)$, to the right until they collide with $S_{b a}(v-a)$ and cancel due to (3.16). The overall effect is an exchange of blue/black dashed lines as well as of the parameters $u$ and $v$ (right picture).
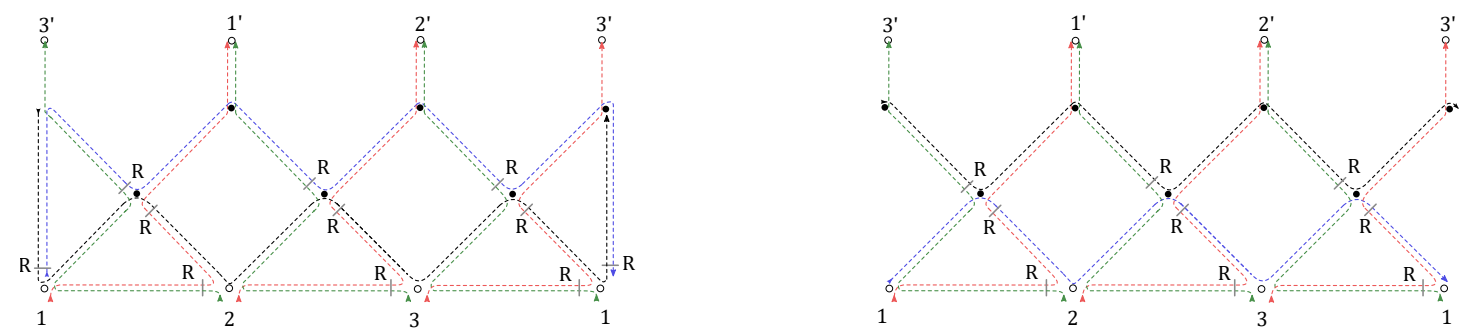

Finally, it is enough to perform the inverse of the first step, integrating the star integrals into triangles $\mathcal{R}^{+}(u)$, recovering the r.h.s. of (4.22)

In order to prove the integrability of the model, we finally observe that the property (3.20) and the definition (4.20) imply that the hermitean conjugate of $t_{+}^{(a)}(u)$ is an integral operator with the same kernel of $t_{-}^{(a)}\left(u^{*}\right)$, apart from the flow of auxiliary space $\boldsymbol{\sigma}$, $\overline{\boldsymbol{\sigma}}$ and the relative position of $\mathbf{R}(u)$ matrices which is the opposite. In diagrammatic form this amounts to

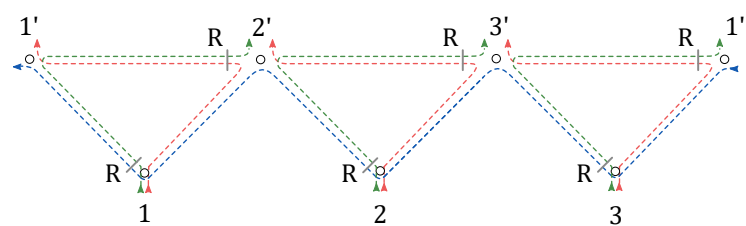

and from this observation it follows that the proof of (16) can be repeated step-by-step in order to proof that

$$
t_{ \pm}^{(a)}(u) t_{ \pm}^{(b)}(v)^{\dagger}=t_{ \pm}^{(b)}(v)^{\dagger} t_{ \pm}^{(a)}(u),
$$

with the only difference that the interchange relation involved instead of figure 9 is the one of figure 10. The equations (4.22) and (4.23) show that the "half" transfer matrices $t_{ \pm}^{(a)}(u)$ are an alternative way to define the same integrable model, as their Taylor expansion in $u$ generates two families of conserved charges. 

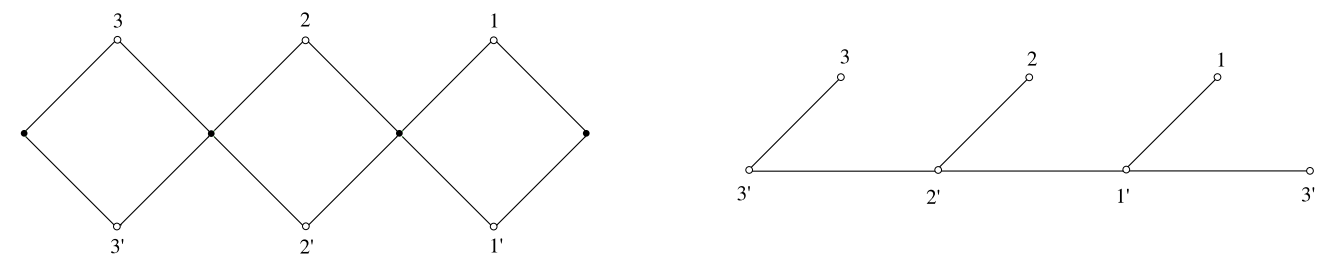

Figure 17. Left: integral kernel of the transfer matrix operator $t^{(a)}(u)$ for a spin chain of length $L=3$, for auxiliary and physical spaces in the representation of zero spins $\ell_{a}=\ell_{k}=0$ and $\dot{\ell}_{a}=\dot{\ell}_{k}=0$ and $\Delta_{k}=\Delta_{a}=1$. Right: at the point $u=-1$ and $\theta=0$ (or, equivalently, $u \rightarrow-1 / 2$ and $\theta=-\Delta / 2=-1 / 2$ ) the transfer matrix kernel reduces to the graph-building operator $\hat{B}$ for the planar Feynman integrals of the bi-scalar Fishnet theory (4.24).

\subsection{Integrable correlators in conformal field theories}

In the papers $[1,24,34]$, the authors dealt with the homogeneous chain of particles in the scalar representation $\left(\Delta_{k}, \ell_{k}, \dot{\ell}_{k}\right)=(\Delta, 0,0)$ and for an auxiliary space in the same representation. It was observed that for $\Delta=1$ at the point $u=-1$ and $\theta=0$ the transfer matrix operator $t^{(a)}(u)$ reduces to the graph-building operator for a class of planar Feynman diagrams with regular square-lattice topology (see figure 17), and its spectral problem was directly related to the spectra of scaling dimensions of the single-trace vacua $\operatorname{Tr}\left[\phi_{1}^{L}\right](x)$ of the conformal Fishnet theory (FCFT) introduced in [1]

$$
\mathcal{L}_{\phi}=\frac{N}{2} \operatorname{Tr}\left(\partial^{\mu} \phi_{1}^{\dagger} \partial_{\mu} \phi^{1}+\partial^{\mu} \phi_{2}^{\dagger} \partial_{\mu} \phi^{2}+2 \xi^{2} \phi_{1}^{\dagger} \phi_{2}^{\dagger} \phi^{1} \phi^{2}\right)
$$

where $\phi_{k}$ are $N \times N$ complex scalar fields in the adjoint representation of $\mathrm{SU}(N)$. In fact, the scaling behaviour of the two point functions of vacua at $L>2$ at finite coupling is captured by a geometric series

$$
\lim _{y \rightarrow \infty}\left\langle\operatorname{Tr}\left[\phi_{1}^{L}\right](x) \operatorname{Tr}\left[\phi_{1}^{L}\right]^{\dagger}(y)\right\rangle \propto\left\langle x\left|\frac{1}{1-\xi^{2 L} \hat{B}}\right| 1\right\rangle, \quad|x\rangle=\prod_{k=1}^{L} \delta^{(4)}\left(x_{k}-x\right),|1\rangle=1,
$$

where $\hat{B}$ is the operator defined by the limit $u \rightarrow-1$ of the transfer matrix. The Feynman integrals contributing to the correlator in perturbation theory have a simple square-lattice fishnet topology and they are built by iterative composition of the operator $\xi^{2 L} \hat{B}$, which therefore plays the role of integral Bethe-Salpeter kernel of the correlator, and the geometric series follows from the re-summation of all perturbative orders. The equation (4.25) is a statement of the quantum integrability of the FCTF vacua, as it maps the spectral problem for the scaling dimensions to an integrable quantum chain of particles. The above analysis can be extended to other operators than the vacua, for instance the ones obtained by insertion of an excitation $\phi_{2}$ upon the vacuum of $L>1$ fields $\phi_{1}$

$$
\mathcal{O}_{L}^{\prime}(x)=\operatorname{Tr}\left[\phi_{1}^{L} \phi_{2}\right](x),
$$

Its two-point function has a scaling behaviour captured by the expression

$$
\lim _{y \rightarrow \infty}\left\langle\mathcal{O}_{L}^{\prime}(x) \mathcal{O}_{L}^{\prime}(y)^{\dagger}\right\rangle \propto \sum_{k=0}^{L-1} \frac{\xi^{2 k}}{\left(4 \pi^{2}\right)^{2 k}}\left\langle x\left|\hat{B}_{k}^{\prime} \frac{1}{1-\xi^{2 L} \hat{B}_{L}^{\prime}}\right| 1\right\rangle,
$$



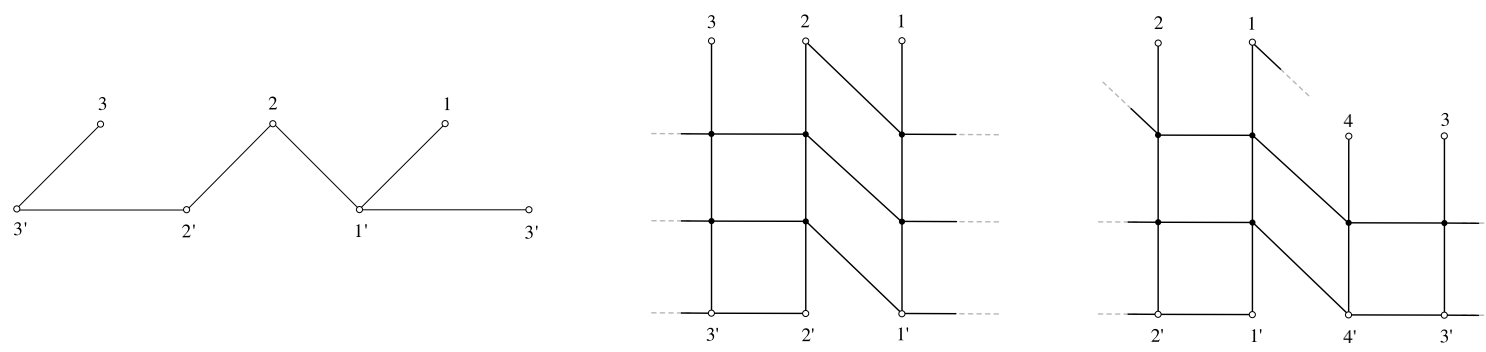

Figure 18. Left: reduction at the point $u \rightarrow-1 / 2$ of the kernel of the transfer matrix operator $t^{(a)}(u)$ for a spin chain of length $L=3$, for auxiliary and physical spaces in the representation of zero spins $\ell_{a}=\ell_{k}=0, \dot{\ell}_{a}=\dot{\ell}_{k}=0$ and scaling dimensions $\Delta_{1,3}=\Delta_{a}=1$ and $\Delta_{2}=2$. Center: the iterative composition of the reduced transfer matrix is the graph-builder of the bulk of Feynman integrals contributing to the two point function of $\operatorname{Tr}\left[\phi_{1}^{2} \phi_{2}\right]$ Right: a possible choice of boundary condition for the graphs of $\operatorname{Tr}\left[\phi_{1}^{3} \phi_{2}\right]$, corresponding to the term $k=2$ in (4.27). The first operator from the top of the graph is $\hat{B}_{2}^{\prime}$ while the second and third are $\hat{B}_{4}^{\prime}$ and add both a wrapping around the graph.

where here the graph-builder $\hat{B}_{L}^{\prime}$ is the limit $u \rightarrow-1 / 2$ of the transfer matrix of a spinless inhomogeneous chain of $L$ particles, out of which $L-1$ have dimension $\Delta=1(\theta=-1 / 2)$ and stand for a field $\phi_{1}$, and one has dimension $\Delta=2(\theta=-1)$ and stands for the pair of fields $\phi_{1} \phi_{2}$ (see figure 18). The sum over $k$ in (4.27) involves the operator $\hat{B}_{k}^{\prime}$ acting on the first $k$ sites starting from the "magnonic one" $\Delta=2$, and accounts for the different boundary conditions of the graphs, while the geometric series in $\hat{B}_{L}^{\prime}$ captures the graphs bulk. The class of Feynman integrals entering the perturbative expansion of such correlators is depicted in figure 18 together with the graph-building kernel, so to make clear the origin of the geometric series and boundary terms in (4.27). The arguments of the previous paragraphs can be repeated in full generality for every local operator of the bi-scalar theory (4.24), by an appropriate choice of the representations in the physical spaces of the chain and of inhomogeinities parameters. ${ }^{4}$ Similarly, one can realize via a conformal chain any other correlator of the theory, since the bulk of Feynman integrals is a square-lattice and the only leftover freedom is the choice of boundary conditions for the quantum magnet - imposed by the choice of the correlator (see for example the four-point correlators $[4,36]$ corresponding to an open conformal chain, or $n$-point correlators analyzed in [37, 38]).

In the previous section we have showed that the transfer matrices $t^{(a)}(u)$ can be worked out in the language of Feynman integrals for any unitary irreps $\mathbb{V}_{i}$ and $\mathbb{V}_{a}$ of the conformal group. Therefore, $t^{(a)}(u)$ can be regarded as the graph-building operator for a class of planar Feynman diagrams with square lattice topology, which we can dub spinning (for some $\left(\ell_{k}, \dot{\ell_{k}}\right) \neq(0,0)$ ) and inhomogeneous (for $\mathbb{V}_{k} \neq \mathbb{V}_{j}$ ) fishnets, represented in figure 19. The quartic vertex of the lattice is scale invariant and mixes the $\mathrm{SU}(2)$ spinor indices of the incoming/outcoming fields by means of the fused $\mathbf{R}$-matrix (2.19).

The graph-building operators $t^{(a)}(u)$ of the spinning inhomogeneous fishnet for an appropriate choice of physical and auxiliary space representations describe other correlators in the broader context of chiral (a.k.a. generalized fishnet) CFTs, proving such correlators

\footnotetext{
${ }^{4}$ For a complete analysis of how to realize FCTF two-point functions starting from a spin-less conformal spin chain with inhomogeinities the reader can refer to [35].
} 

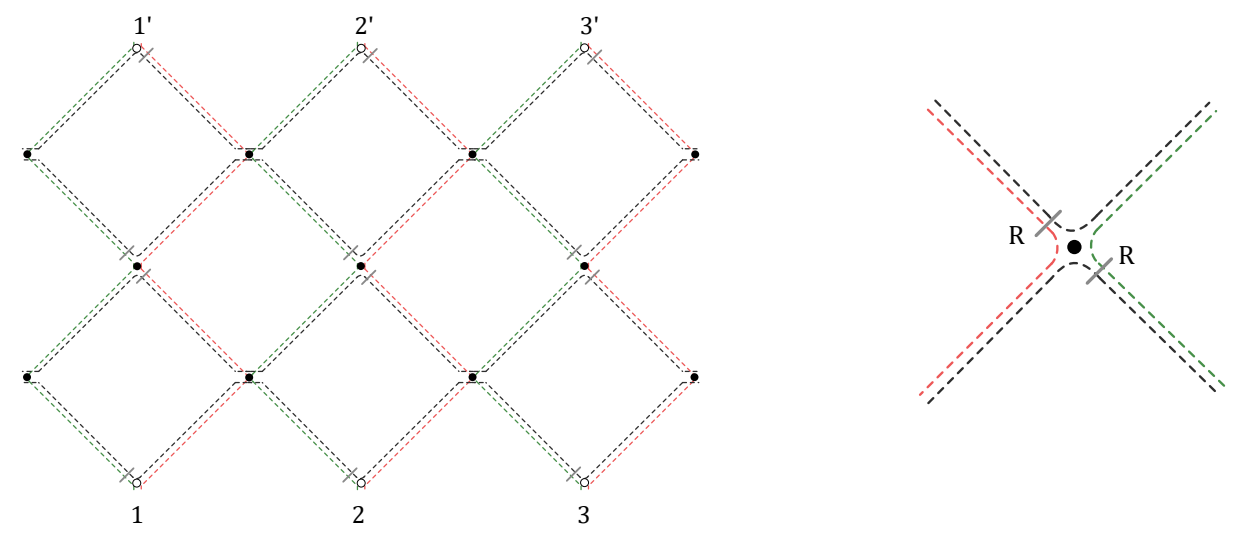

Figure 19. Left: spinning inhomogeneous fishnet Feynman diagram: any red line and green line carries a different Weyl spinor representation $\left(\Delta_{k}, \ell_{k}, 0\right),\left(\Delta_{k}, 0, \dot{\ell}_{k}\right)$, while black lines are associated to the auxiliary space representations and their representation is set to $\left(\Delta_{a}, \ell_{a}, 0\right)$ and $\left(\Delta_{a}, 0, \dot{\ell}_{a}\right)$. Each integration point on the left is identified with the corresponding on the right, so the graph is wrapped on a cylinder. Right: quartic vertex of the planar lattice.

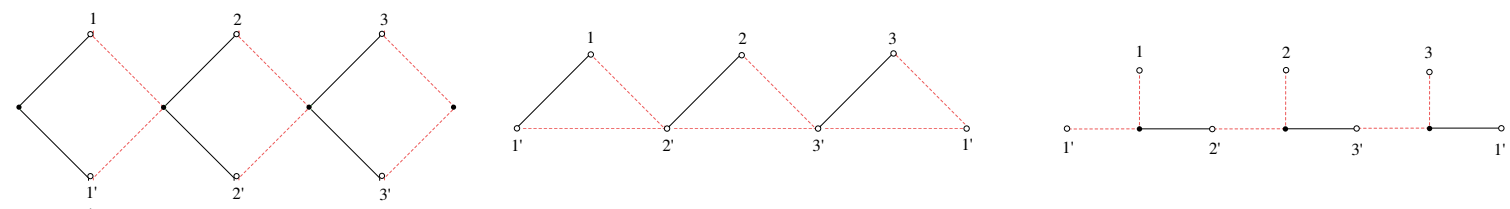

Figure 20. Left: integral kernel of the transfer matrix operator $t^{(a)}(u)$ for a spin chain of length $L=$ 3 , for spinless auxiliary space $\ell_{a}=\dot{\ell}_{a}=0$ and physical spaces in the Weyl fermion representation $(3 / 2,0,1)$. Center: the transfer matrix kernel at $u \rightarrow-5 / 4$ and $\theta=0$ takes the shape of a triangular lattice wrapped onto a cylinder. Right: by means of star-triangle duality the triangular lattice can be re-written as an hexagonal lattice of Yukawa vertices, where fermions propagate along the cylinder and scalar around it.

to be integrable from a spin chain picture. For example, an homogeneous spinning fishnet of Weyl fermions describes the strong deformation limit defined in [19] of the $\mathcal{N}=2 \mathrm{SYM}$ planar two point correlators among the fermionic vacua

$$
\mathcal{O}_{a_{1} \ldots a_{L}}(x)=\operatorname{Tr}\left[\left(\lambda_{1}\right)_{a_{1}} \cdots\left(\lambda_{1}\right)_{a_{L}}\right](x), a_{j}=1,2 .
$$

In this case the Feynman integrals of the perturbative expansion are described by an hexagonal lattice of Yukawa vertices, where fermions propagate along between the two operators (physical space), and scalar fields form wrappings in the orthogonal direction (auxiliary space), therefore the spin chain is defined by $\left(\Delta_{k}, \ell_{k}, \dot{\ell}_{k}\right)=(3 / 2,0,1)$ and $\left(\Delta_{a}, \ell_{a}, \dot{\ell}_{a}\right)=(1,0,0)$. Another class of integrable correlators described by honeycomb Feynman integrals - though with different boundary conditions - are the bosonic vacua $\operatorname{Tr}\left[\phi^{L}\right](x)$ in the same planar theory. In this case the fermions propagate around forming wrappings (i.e. auxiliary space), and scalar fields propagating between the two operators (physical space). Therefore one deals — as for the FCFT (4.24) — with the scalar homogeneous model $\left(\Delta_{k}, \ell_{k}, \dot{\ell}_{k}\right)=(1,0,0)$, but the graph-builder is a transfer matrix with fermionic auxiliary space $\left(\Delta_{a}, \ell_{a}, \dot{\ell}_{a}\right)=(3 / 2,0,1)$. Furthermore, the extension to inhomo- 

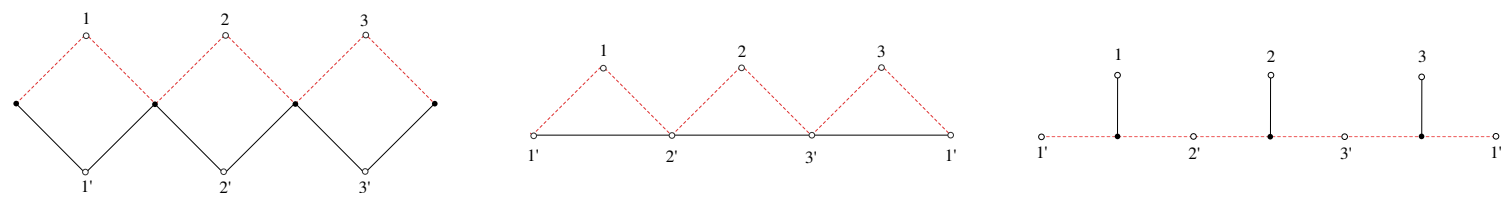

Figure 21. Left: integral kernel of the transfer matrix operator $t^{(a)}(u)$ for a spin chain of length $L=3$, for auxiliary space in the Weyl fermion representation $(3 / 2,0,1)$ and physical spaces in the scalar representation $(1,0,0)$. Center: the transfer matrix kernel at $u=-5 / 4$ takes the shape of a triangular lattice wrapped onto a cylinder. Right: by means of star-triangle duality the triangular lattice can be re-written as an hexagonal lattice, with fermions propagating in angular direction and scalars along the cylinder.
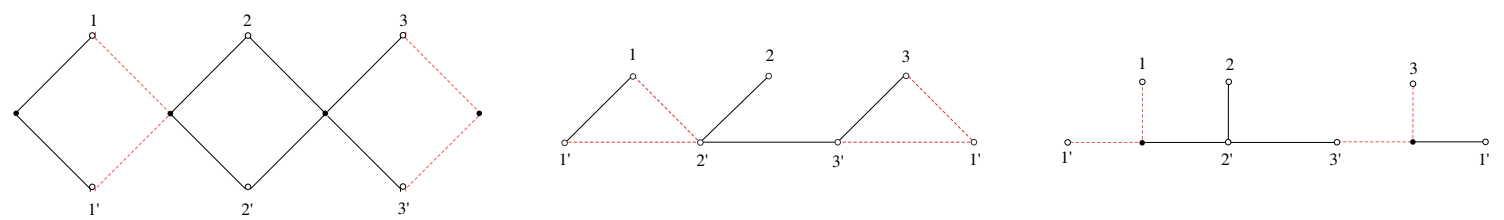

Figure 22. Left: integral kernel of the transfer matrix operator $t^{(a)}(u)$ for a spin chain of length $L=3$, for scalar auxiliary space $\ell_{a}=\dot{\ell}_{a}=0$ and inhomogeneous representation in physical spaces: scalar $(1,0,0)$ or Weyl fermion $(3 / 2,0,1)$, with inhomogeinities $\theta=-\Delta / 2$ according to (4.10). Center: reduction of the kernel at the point $u \rightarrow-1 / 2$. Right: by star-triangle duality the reduced kernel can be written as the graph building operator for a planar Feynman diagram wrapped onto a cylinder, whose topology mixes square-lattice and Yukawa hexagonal lattice.

geneous chain allows to mix scalars $(1,0,0)$ and Weyl fermions $(3 / 2,0,1)$ in the physical space, describing new classes of planar Feynman integrals. The transfer matrix of such a chain for $L$ scalar particles and $N$ fermionic ones is the graph builder of Feynman diagrams that mix the topology of square and honeycomb lattice. Such a class of integrals describes completely the perturbation theory of two-point planar correlators of a single-trace operator

$$
O_{L, a_{1} \ldots a_{N}}(x)=\operatorname{Tr}\left[\phi_{1}^{L}\left(\psi_{1}\right)_{a_{1}} \cdots\left(\psi_{1}\right)_{a_{N}}\right](x)+\text { (permutations), }
$$

in the double-scaling limit of $\gamma_{i}$-deformed $\mathcal{N}=4$ SYM theory [16]:

$$
\gamma_{1}, \gamma_{2} \rightarrow-i \infty, \gamma_{3} \rightarrow i \infty, g \rightarrow 0 \mid g^{2} e^{i \gamma_{1}}=\xi_{1}^{2}, g^{2} e^{i \gamma_{2}}=\xi_{2}^{2} .
$$

Thus, by the same arguments of the previous paragraphs, we can map the spectrum of the theory's scaling dimensions to the diagonalization of transfer matrices of the inhomogeneous spinning integrable chain with representation $(1,0,0), \theta=-1 / 2$ and $(3 / 2,0,1), \theta=-3 / 4$.

\section{Outlooks}

The methods developed in this paper shall find application to multiple open problems. The generalization of star-triangle duality to any spinning propagator is a crucial step in order to mimic the $2 d$ techniques of [15] and construct the transformation to separated variables [39] for the integrable model of section 4 . Indeed, the attentive reader has certainly noticed a striking similarity between the integral kernels of figure 16 and the eigenfunctions 
of the $B(u)$ operator in the $\mathrm{SL}(2, \mathbb{C})$ chain. The importance of constructing the separation of variables $(\mathrm{SoV})$ transform in the $\mathrm{SO}(1,5)$ chain is multiple: first the recent achievements of [40-49] would be extended to the principal series representations - that appears in any operator product expansion in a CFT $[14,50]$. Moreover as a consequence of the considerations of section 4.1 the SoV would lead to an interesting example of direct derivation of a quantum spectral curve in the Fishnet and chiral conformal field theories [1, 16, 18, 19].

Another captivating model to study by these new means is the open boundary version of the inhomogeneous spinning fishnet. In analogy with the computation of Basso-Dixon diagrams $[4,5,25]$, a general inhomogeneous and spinning version of the fishnet lattice can be used to compute 4-point correlators in the double-scaling (DS) limit of $\mathcal{N}=4$ SYM $[1,17]$, a richer theory than the bi-scalar fishnet that is a promising starting point to understand integrable structures in the undeformed theory. Similar considerations apply to higher-point correlators, where the technique of tessellations [51-55] may be repeated with a direct approach - the computation of Feynman integrals - in various DS limits of the SYM theory. This perspective is particularly appealing as it promises to shed light on the relation between the $\mathrm{SoV}$ and the computation of hexagon form factors.

Finally, the formulation of a fishnet theory in any even space-time dimension $d[23,26]$ leaves the question open about how to extend the star-triangle dualities, diagrams computation, separation of variables, from $\mathrm{SL}(2, \mathbb{C}) \simeq \mathrm{SO}(1,3)$ and $\mathrm{SO}(1,5)$ to the conformal spin chain $\mathrm{SO}(1, d+1)$.

\section{Acknowledgments}

We thank A.N. Manashov and especially G. Ferrando for careful reading and useful comments on the manuscript. The work of S.D. was supported by the Russian Science Foundation project No 19-11-00131. Research at the Perimeter Institute is supported in part by the Government of Canada through NSERC and by the Province of Ontario through MRI. This work was additionally supported by a grant from the Simons Foundation (Simons Collaboration on the Nonperturbative Bootstrap).

Open Access. This article is distributed under the terms of the Creative Commons Attribution License (CC-BY 4.0), which permits any use, distribution and reproduction in any medium, provided the original author(s) and source are credited.

\section{References}

[1] O. Gürdoğan and V. Kazakov, New integrable $4 D$ quantum field theories from strongly deformed planar $N=4$ supersymmetric Yang-Mills theory, Phys. Rev. Lett. 117 (2016) 201602 [Addendum ibid. 117 (2016) 259903] [arXiv: 1512.06704] [INSPIRE].

[2] A.P. Isaev, Multiloop Feynman integrals and conformal quantum mechanics, Nucl. Phys. B 662 (2003) 461 [hep-th/0303056] [inSPIRE].

[3] D. Chicherin, S. Derkachov and A.P. Isaev, Conformal group: R-matrix and star-triangle relation, JHEP 04 (2013) 020 [arXiv: 1206.4150] [INSPIRE]. 
[4] S. Derkachov and E. Olivucci, Exactly solvable magnet of conformal spins in four dimensions, Phys. Rev. Lett. 125 (2020) 031603 [arXiv: 1912.07588] [INSPIRE].

[5] S. Derkachov and E. Olivucci, Exactly solvable single-trace four point correlators in $\chi \mathrm{CFT}_{4}$, JHEP 02 (2021) 146 [arXiv:2007.15049] [INSPIRE].

[6] A.P. Isaev, Operator approach to analytical evaluation of Feynman diagrams, Phys. Atom. Nucl. 71 (2008) 914 [arXiv:0709.0419] [INSPIRE].

[7] A.N. Vasiliev, Y.M. Pismak and Y.R. Khonkonen, $1 / N$ expansion: calculation of the exponent $\eta$ in the order $1 / N^{3}$ by the conformal bootstrap method, Theor. Math. Phys. 50 (1982) 127 [Teor. Mat. Fiz. 50 (1982) 195] [INSPIRE].

[8] A.N. Vasiliev, Y.M. Pismak and Y.R. Khonkonen, $1 / N$ expansion: calculation of the exponents $\eta$ and $\nu$ in the order $1 / N^{2}$ for arbitrary number of dimensions, Theor. Math. Phys. 47 (1981) 465 [Teor. Mat. Fiz. 47 (1981) 291] [INSPIRE].

[9] D.I. Kazakov, Calculation of Feynman integrals by the method of 'uniqueness', Theor. Math. Phys. 58 (1984) 223 [Teor. Mat. Fiz. 58 (1984) 343] [INSPIRE].

[10] D.I. Kazakov, Multiloop calculations: method of uniqueness and functional equations, Teor. Mat. Fiz. 62 (1984) 127 [Teor. Mat. Fiz. 62 (1984) 127] [INSPIRE].

[11] D.I. Kazakov, The method of uniqueness, a new powerful technique for multiloop calculations, Phys. Lett. B 133 (1983) 406 [InSPIRE].

[12] M. Preti, STR: a Mathematica package for the method of uniqueness, Int. J. Mod. Phys. C 31 (2020) 2050146 [arXiv:1811.04935] [INSPIRE].

[13] M. Preti, The game of triangles, J. Phys. Conf. Ser. 1525 (2020) 012015 [arXiv: 1905.07380] [INSPIRE].

[14] V.K. Dobrev, G. Mack, V.B. Petkova, S.G. Petrova and I.T. Todorov, Harmonic analysis on the $n$-dimensional Lorentz group and its application to conformal quantum field theory, Lect. Notes Phys. 63 (1977) 059 [INSPIRE].

[15] S.E. Derkachov, G.P. Korchemsky and A.N. Manashov, Noncompact Heisenberg spin magnets from high-energy QCD: 1 . Baxter $Q$ operator and separation of variables, Nucl. Phys. B 617 (2001) 375 [hep-th/0107193] [INSPIRE].

[16] J. Caetano, O. Gürdoğan and V. Kazakov, Chiral limit of $N=4 S Y M$ and ABJM and integrable Feynman graphs, JHEP 03 (2018) 077 [arXiv: 1612.05895] [INSPIRE].

[17] V. Kazakov, Quantum spectral curve of $\gamma$-twisted $N=4$ SYM theory and fishnet CFT, Rev. Math. Phys. 30 (2018) 1840010.

[18] V. Kazakov, E. Olivucci and M. Preti, Generalized fishnets and exact four-point correlators in chiral $\mathrm{CFT}_{4}$, JHEP 06 (2019) 078 [arXiv: 1901.00011] [INSPIRE].

[19] A. Pittelli and M. Preti, Integrable fishnet from $\gamma$-deformed $N=2$ quivers, Phys. Lett. $B$ 798 (2019) 134971.

[20] L.N. Lipatov, High-energy asymptotics of multicolor QCD and two-dimensional conformal field theories, Phys. Lett. B 309 (1993) 394 [INSPIRE].

[21] L.N. Lipatov, Asymptotic behavior of multicolor QCD at high energies in connection with exactly solvable spin models, JETP Lett. 59 (1994) 596 [Pisma Zh. Eksp. Teor. Fiz. 59 (1994) 571] [hep-th/9311037] [INSPIRE]. 
[22] L.D. Faddeev and G.P. Korchemsky, High-energy QCD as a completely integrable model, Phys. Lett. B 342 (1995) 311 [hep-th/9404173] [INSPIRE].

[23] V. Kazakov and E. Olivucci, Biscalar integrable conformal field theories in any dimension, Phys. Rev. Lett. 121 (2018) 131601 [arXiv:1801.09844] [INSPIRE].

[24] N. Gromov, V. Kazakov, G. Korchemsky, S. Negro and G. Sizov, Integrability of conformal fishnet theory, JHEP 01 (2018) 095 [arXiv: 1706. 04167] [INSPIRE].

[25] S. Derkachov, V. Kazakov and E. Olivucci, Basso-Dixon correlators in two-dimensional fishnet CFT, JHEP 04 (2019) 032 [arXiv:1811.10623] [INSPIRE].

[26] B. Basso, G. Ferrando, V. Kazakov and D.-L. Zhong, Thermodynamic Bethe ansatz for biscalar conformal field theories in any dimension, Phys. Rev. Lett. 125 (2020) 091601 [arXiv: 1911.10213] [INSPIRE].

[27] I.M. Gelfand and G.E. Shilov, Generalized functions, volume I, AMS Chelsea Publishing, Academic Press, New York, NY, U.S.A. (1964).

[28] G.M. Sotkov and R.P. Zaikov, Conformal invariant two point and three point functions for fields with arbitrary spin, Rept. Math. Phys. 12 (1977) 375 [INSPIRE].

[29] P.P. Kulish, N.Y. Reshetikhin and E.K. Sklyanin, Yang-Baxter equation and representation theory. 1, Lett. Math. Phys. 5 (1981) 393 [InSPIRE].

[30] P.P. Kulish and E.K. Sklyanin, Quantum spectral transform method. Recent developments, Lect. Notes Phys. 151 (1982) 61 [INSPIRE].

[31] L.D. Faddeev, How algebraic Bethe ansatz works for integrable model, in Les Houches school of physics: astrophysical sources of gravitational radiation, (1996), pg. 149 [hep-th/9605187] [INSPIRE].

[32] P.P. Kulish and E.K. Sklyanin, On the solution of the Yang-Baxter equation, J. Sov. Math. 19 (1982) 1596 [Zap. Nauchn. Semin. 95 (1980) 129] [InSPIRE].

[33] V.O. Tarasov, L.A. Takhtajan and L.D. Faddeev, Local Hamiltonians for integrable quantum models on a lattice, Theor. Math. Phys. 57 (1983) 1059 [Teor. Mat. Fiz. 57 (1983) 163] [INSPIRE].

[34] N. Gromov, V. Kazakov and G. Korchemsky, Exact correlation functions in conformal fishnet theory, JHEP 08 (2019) 123 [arXiv: 1808. 02688] [INSPIRE].

[35] N. Gromov and A. Sever, The holographic dual of strongly $\gamma$-deformed N $=4 S Y M$ theory: derivation, generalization, integrability and discrete reparametrization symmetry, JHEP 02 (2020) 035 [arXiv: 1908.10379] [InSPIRE].

[36] B. Basso and L.J. Dixon, Gluing ladder Feynman diagrams into fishnets, Phys. Rev. Lett. 119 (2017) 071601 [arXiv:1705.03545] [INSPIRE].

[37] D. Chicherin, V. Kazakov, F. Loebbert, D. Müller and D.-L. Zhong, Yangian symmetry for fishnet Feynman graphs, Phys. Rev. D 96 (2017) 121901 [arXiv: 1708.00007] [INSPIRE].

[38] D. Chicherin, V. Kazakov, F. Loebbert, D. Müller and D.-L. Zhong, Yangian symmetry for bi-scalar loop amplitudes, JHEP 05 (2018) 003 [arXiv:1704.01967] [INSPIRE].

[39] E.K. Sklyanin, Separation of variables - new trends, Prog. Theor. Phys. Suppl. 118 (1995) 35 [solv-int/9504001] [INSPIRE]. 
[40] N. Gromov, F. Levkovich-Maslyuk and P. Ryan, Determinant form of correlators in high rank integrable spin chains via separation of variables, JHEP 05 (2021) 169 [arXiv: 2011.08229] [INSPIRE].

[41] N. Gromov, F. Levkovich-Maslyuk, P. Ryan and D. Volin, Dual separated variables and scalar products, Phys. Lett. B 806 (2020) 135494 [arXiv:1910.13442] [INSPIRE].

[42] A. Cavaglià, N. Gromov and F. Levkovich-Maslyuk, Separation of variables and scalar products at any rank, JHEP 09 (2019) 052 [arXiv: 1907.03788] [INSPIRE].

[43] N. Gromov, F. Levkovich-Maslyuk and G. Sizov, New construction of eigenstates and separation of variables for $\mathrm{SU}(N)$ quantum spin chains, JHEP 09 (2017) 111 [arXiv: 1610.08032] [INSPIRE].

[44] P. Ryan and D. Volin, Separation of variables for rational $g l(n)$ spin chains in any compact representation, via fusion, embedding morphism and Bäcklund flow, Commun. Math. Phys. 383 (2021) 311 [arXiv: 2002.12341] [INSPIRE].

[45] P. Ryan and D. Volin, Separated variables and wave functions for rational gl $(N)$ spin chains in the companion twist frame, J. Math. Phys. 60 (2019) 032701 [arXiv:1810.10996] [INSPIRE].

[46] J.M. Maillet and G. Niccoli, On quantum separation of variables, J. Math. Phys. 59 (2018) 091417 [arXiv: 1807.11572] [INSPIRE].

[47] J.M. Maillet, G. Niccoli and L. Vignoli, On scalar products in higher rank quantum separation of variables, SciPost Phys. 9 (2020) 086 [arXiv:2003.04281] [INSPIRE].

[48] J.M. Maillet and G. Niccoli, On quantum separation of variables beyond fundamental representations, SciPost Phys. 10 (2021) 026 [arXiv: 1903.06618] [INSPIRE].

[49] S.E. Derkachov and P.A. Valinevich, Separation of variables for the quantum $\mathrm{SL}(3, C)$ spin magnet: eigenfunctions of Sklyanin B-operator, J. Math. Sci. 242 (2019) 658 [Zap. Nauchn. Semin. 473 (2018) 110] [arXiv: 1807.00302] [INSPIRE].

[50] P. Di Francesco, P. Mathieu and D. Senechal, Conformal field theory, Springer-Verlag, New York, NY, U.S.A. (1997) [INSPIRE].

[51] B. Basso, S. Komatsu and P. Vieira, Structure constants and integrable bootstrap in planar $N=4 S Y M$ theory, arXiv:1505.06745 [INSPIRE].

[52] T. Fleury and S. Komatsu, Hexagonalization of correlation functions, JHEP 01 (2017) 130 [arXiv: 1611.05577] [INSPIRE].

[53] T. Fleury and S. Komatsu, Hexagonalization of correlation functions. Part II. Two-particle contributions, JHEP 02 (2018) 177 [arXiv:1711.05327] [INSPIRE].

[54] B. Eden and A. Sfondrini, Tessellating cushions: four-point functions in $N=4$ SYM, JHEP 10 (2017) 098 [arXiv:1611.05436] [INSPIRE].

[55] B. Basso, J. Caetano and T. Fleury, Hexagons and correlators in the fishnet theory, JHEP 11 (2019) 172 [arXiv:1812.09794] [INSPIRE]. 